OU-HET-946

\title{
Atiyah-Patodi-Singer index from the domain-wall fermion Dirac operator
}

\author{
Hidenori Fukaya, Tetsuya Onogi, and Satoshi Yamaguchi \\ Department of Physics, Osaka University, Toyonaka 560-0043, Japan
}

\begin{abstract}
The Atiyah-Patodi-Singer(APS) index theorem attracts attention for understanding physics on the surface of materials in topological phases. The mathematical set-up for this theorem is, however, not directly related to the physical fermion system, as it imposes on the fermion fields a non-local boundary condition known as the "APS boundary condition" by hand, which is unlikely to be realized in the materials. In this work, we attempt to reformulate the APS index in a "physicistfriendly" way for a simple set-up with $U(1)$ or $S U(N)$ gauge group on a flat four-dimensional Euclidean space. We find that the same index as APS is obtained from the domain-wall fermion Dirac operator with a local boundary condition, which is naturally given by the kink structure in the mass term. As the boundary condition does not depend on the gauge fields, our new definition of the index is easy to compute with the standard Fujikawa method.
\end{abstract}




\section{INTRODUCTION}

The Atiyah-Singer(AS) index theorem [1, 2] on a four-dimensional closed Euclidean manifold $X$ with flat metric is given by

$$
n_{+}-n_{-}=\frac{1}{32 \pi^{2}} \int_{X} d^{4} x \epsilon_{\mu \nu \rho \sigma} \operatorname{tr}_{c} F^{\mu \nu} F^{\rho \sigma},
$$

where $n_{ \pm}$denotes the number of \pm chiral zero modes of the Dirac operator $D$, and $F_{\mu \nu}$ is the field strength of $S U(N)$ or $U(1)$ gauge fields, for which the $\operatorname{trace} \operatorname{tr}_{c}$ is taken. This theorem is well known in physics [3] and can be easily understood by the so-called Fujikawa method [4],

$$
n_{+}-n_{-}=\lim _{t \rightarrow 0} \operatorname{Tr} \gamma_{5} e^{-t D^{\dagger} D}=\lim _{t \rightarrow 0} \int d^{4} x \operatorname{tr}_{s, c} \sum_{n} \phi_{n}^{\dagger}(x) \gamma_{5} e^{-t D^{\dagger} D} \phi_{n}(x),
$$

where the trace Tr is taken over space-time coordinates, spinor and color indices, while $\operatorname{tr}_{s, c}$ means that for spinor and color indices only. The exponential factor $e^{-t D^{\dagger} D}$ regularizes the trace (heat kernel regularization). Taking the simple plane waves for the complete set $\phi_{n}(x)$, the right-hand side of Eq. (1) is obtained as the leading contribution in the $t$ expansion, which survives the $t \rightarrow 0$ limit. Note that the left-hand side is unchanged even when $t$ is finite, since every non-zero $D^{\dagger} D$ eigenmode makes a pair with its opposite chirality, and does not contribute to the trace.

Next let us consider a manifold extending only in the region $x_{4}>0$, whose boundary at $x_{4}=0$ forms a flat three-dimensional manifold $Y$. Atiyah, Patodi and Singer (APS) [5] (see also [6, 7]) showed that imposing a non-trivial boundary condition (APS boundary condition) on the Dirac operator, the index is given by

$$
\lim _{t \rightarrow 0} \operatorname{Tr} \gamma_{5} e^{-t D^{\dagger} D}=\frac{1}{32 \pi^{2}} \int_{x_{4}>0} d^{4} x \epsilon_{\mu \nu \rho \sigma} \operatorname{tr}_{c} F^{\mu \nu} F^{\rho \sigma}-\frac{\eta\left(i D^{3 \mathrm{D}}\right)}{2},
$$

where $i D^{3 \mathrm{D}}$ is the three-dimensional Dirac operator on $Y$, and $\eta(H)$ is the so-called $\eta$ invariant which is the (regularized) number of non-negative modes subtracted by the number of negative modes of a Hermitian operator $H$. An explicit definition is, for example, given by the (generalized) $\zeta$-function regularization as

$$
\eta(H)=\lim _{s \rightarrow 0} \sum_{\lambda \neq 0} \frac{\lambda}{|\lambda|^{1+s}}+h
$$


where $\lambda$ denotes the eigenvalue of $H$, and $h$ is the number of zero modes of $H$. Because of the regularization, $\eta\left(i D^{3 \mathrm{D}}\right)$ is non-integer in general. In fact, it is equivalent to the Chern-Simons (CS) term

$$
\begin{aligned}
\frac{\eta\left(i D^{3 \mathrm{D}}\right)}{2} & =\frac{C S}{2 \pi} \text { mod integer } \\
C S & \equiv \frac{1}{4 \pi} \int_{Y} d^{3} x \operatorname{tr}_{c}\left[\epsilon_{\nu \rho \sigma}\left(A^{\nu} \partial^{\rho} A^{\sigma}+\frac{2 i}{3} A^{\nu} A^{\rho} A^{\sigma}\right)\right]
\end{aligned}
$$

which precisely cancels the surface contribution in the first term of Eq. (3). Therefore, the total contribution is guaranteed to be an integer.

The APS index theorem describes (a part of) the anomaly descent equations [8-12]. The parity anomaly [13 15] or Chern-Simons term in three dimensions appears as the surface term of the axial $U(1)$ anomaly in the bulk four dimensions. This (parity) anomaly inflow is important to understand the physics of topological insulators [16 25] ${ }^{1}$. The APS theorem indicates that massless edge modes, having parity anomaly, must appear to cancel the parity violation induced by the $U(1)$ anomaly of bulk fermions. For this reason, the APS index theorem attracts attention for understanding physics on the surface of materials in topological phases.

However, the original set-up by APS is not directly related to the physics of topological insulators. APS considered a Dirac operator for massless fermions with a non-local boundary condition called the APS boundary condition, which is introduced in a rather ad hoc way. On the other hand, the fermion in a topological insulator is massive in the bulk, and has a local boundary condition, which keeps the $S O(3)$ (or $S O(2,1)$ in Minkowski space-time) rotational symmetry on the surface. This rotational symmetry is essential for the edge-localized mode to act as a relativistic Dirac fermion, but it is not compatible with the helicity conservation, which is required by the APS condition to keep the bulk fermion massless. In fact, as we explicitly see below, the APS boundary condition allows no edge localized mode to exist in the system, and the eta-invariant appears in an entirely different way from what we expect in the anomaly inflow between bulk and edge modes. In this sense, the fact that the APS index describes the anomaly inflow of topological materials is a coincidence, since the original mathematical setup by APS is nothing to do with the physical fermion system.

1 This work is motivated by recent developments in regularization of chiral fermion using domain-wall fermion formalism, where the gauge anomaly inflow is manifest [26 29]. 
The goal of this work is to reformulate the APS index in a "physicist-friendly" way, as was done by Fujikawa for the AS index on closed manifolds. We propose a new index for a fermion Dirac operator with a mass term having a kink structure, which provides a good model to describe the fermions in topological phases. We find that this index is identical to the APS index, which explains why it appears in the anomaly inflow for the topological insulators. Here, we do not pursue a mathematically precise treatment but a physically sensible way to do the computation. For this purpose, we only consider a simple set-up with the gauge group of $U(1)$ or $S U(N)$ and flat Euclidean metric both in the four-dimensional bulk and at the three-dimensional boundaries. Since the boundary condition does not depend on the gauge fields, our new definition of the index is easy to compute with the standard Fujikawa method.

The rest of this paper is organized as follows. We first review the original APS index theorem in Sec. II] and discuss the problems of the APS boundary condition when we apply it to physics with boundary. Then we consider what is required to realize a more physically natural set-up and show that the domain-wall fermion Dirac operator [30 33] is the best candidate. We show that the same index as APS is obtained through the domain-wall fermion Dirac operator in Sec. III and Sec. IV]. Finally we give a summary and discussion in Sec. V

\section{MASSLESS FERMIONS WITH APS BOUNDARY CONDITION}

In this section we reproduce the results by Atiyah et al. [5] for a much simpler set-up than the original one. We consider a massless Dirac operator in the fundamental representation of $S U(N)$ or $U(1)$ gauge group, taking the $A_{4}=0$ gauge:

$$
D=\gamma_{4}\left(\partial_{4}+A\right)
$$

where $A=\gamma_{4} \sum_{i=1}^{3} \gamma_{i} D_{i}$ with covariant derivative $D_{i}=\partial_{i}+i A_{i}$, being a Hermitian operator. We consider a four-dimensional flat manifold $X$ extending in the region $x_{4}>0$, with a threedimensional boundary $Y$ at $x_{4}=0$.

Then we require the fermion fields, on which $D$ operates, to have a support only from negative eigenfunctions of $A$ at the boundary $x_{4}=0$, which is known as the APS boundary 
condition. Namely, any positive eigenfunction component must vanish:

$$
\left.\frac{A+|A|}{2} \phi\right|_{x_{4}=0}=0
$$

We also consider the opposite case,

$$
\left.\frac{A-|A|}{2} \phi\right|_{x_{4}=0}=0
$$

which we call the anti-APS condition. Since the spectrum of $A$ requires information of gauge fields in the entire $Y$, the APS/anti-APS boundary conditions are non-local. With these non-trivial boundary conditions, the anti-Hermiticity of $D$ is maintained since

$$
\left(\phi_{1}, D \phi_{2}\right) \equiv \int_{X} d^{4} x \phi_{1}^{\dagger}(x) D \phi_{2}(x)=\left.\int_{Y} d^{3} x \phi_{1}^{\dagger}(x) \gamma_{4} \phi_{2}(x)\right|_{x_{4}=0}-\left(D \phi_{1}, \phi_{2}\right)=-\left(D \phi_{1}, \phi_{2}\right)
$$

where we have used the absence of the surface term

$$
\left.\int_{Y} d^{3} x \phi_{1}^{\dagger}(x) \gamma_{4} \phi_{2}(x)\right|_{x_{4}=0}=0
$$

which is a consequence of anti-commutation relation $\left\{\gamma_{4}, A\right\}=0$, so that $\gamma_{4} \phi_{2}$ has a support only from eigenfunction of $A$ with opposite sign of eigenvalues to that of $\phi_{1}$. Therefore, their inner product vanishes.

The anti-Hermiticity of the Dirac operator is not enough to formulate the index theorem since we need twice the operations of $D$ or $D^{\dagger} D=-D^{2}$ to regularize the trace of $\gamma_{5}$. Therefore, we impose the same APS/anti-APS boundary condition also on $D \phi$ (then $D^{n} \phi$ for any $n$ automatically satisfies the same boundary condition).

In this section, it is convenient to take the chiral representation of the $4 \times 4$ gamma matrices or, equivalently, tensor product of $2 \times 2$ matrices as

$$
\begin{aligned}
\gamma_{i=1,2,3} & =\left(\begin{array}{c}
i \sigma_{i} \\
-i \sigma_{i}
\end{array}\right)=-\tau_{2} \otimes \sigma_{i}, \quad \gamma_{4}=\left(\begin{array}{r}
1_{2 \times 2} \\
1_{2 \times 2}
\end{array}\right)=\tau_{1} \otimes 1_{2 \times 2}, \\
\gamma_{5} & =-\gamma_{1} \gamma_{2} \gamma_{3} \gamma_{4}=\left(\begin{array}{cc}
1_{2 \times 2} & \\
-1_{2 \times 2}
\end{array}\right)=\tau_{3} \otimes 1_{2 \times 2},
\end{aligned}
$$

where $1_{2 \times 2}$ denotes the $2 \times 2$ unit matrix, and $\sigma_{i}$ and $\tau_{i}$ denote the Pauli matrices. In this representation, $A$ takes a block-diagonal form

$$
A=\left(\begin{array}{cc}
i D^{3 \mathrm{D}} & \\
& -i D^{3 \mathrm{D}}
\end{array}\right)=\tau_{3} \otimes i D^{3 \mathrm{D}}
$$


where $D^{3 \mathrm{D}}=-\sigma_{i} D^{i}$ denotes the three-dimensional massless Dirac operator. Therefore, positive eigenfunctions of $A$ correspond to positive/negative eigenmodes of $i D^{3 \mathrm{D}}$ for positive/negative chiral modes, respectively. Note that $\gamma_{5}$ commutes with $A$. Therefore, these boundary conditions preserve the helicity of the fermions.

There is a crucial difference between the APS and anti-APS boundary conditions. For simplicity, let us take $A$ as $x_{4}$ independent. Then the anti-APS boundary condition allows an edge-localized zero-mode:

$$
\phi=\phi_{\lambda} e^{-\lambda x_{4}}, \quad D \phi=0
$$

where $\lambda$ and $\phi_{\lambda}$ are a positive eigenvalue and eigenfunction of $A$, respectively, while the APS boundary condition does not allow such zero modes, since the sign flip of the eigenvalue $\lambda$ makes the eigenfunction in Eq. (14) unnormalizable.

\section{A. Computation on a $x_{4}$-independent background}

Following the original paper by APS [5], let us begin with the case where $A$ and therefore its gauge potentials $A_{i}(i=1,2,3)$ have no $x_{4}$ dependence. We take $X$ to be infinitely large in the positive region of $x_{4}$. Namely we consider a flat background in the $x_{4}>0$ region. In this set-up, $F_{4 i}=0$ so that the index theorem should be simply given as

$$
\lim _{t \rightarrow 0} \operatorname{Tr} \gamma_{5} e^{-t D^{\dagger} D}=-\frac{\eta\left(i D^{3 \mathrm{D}}\right)}{2} .
$$

The goal of this subsection is to reproduce this result in our familiar language in physics.

When $A$ has no $x_{4}$ dependence, $D^{\dagger} D$ can be written as

$$
D^{\dagger} D=-\partial_{4}^{2}+A^{2}
$$

which commutes with both $\gamma_{5}$ and $A$. It is, therefore, convenient to consider the eigenvalue problem of $D^{\dagger} D$ by assuming the form of the solution as

$$
\phi_{ \pm}\left(x_{4}\right) \otimes \phi_{\lambda}^{3 \mathrm{D}}(\vec{x}), \quad\left(-\partial_{4}^{2}+\lambda^{2}\right) \phi_{ \pm}\left(x_{4}\right)=\Lambda^{2} \phi_{ \pm}\left(x_{4}\right),
$$

where $\phi_{\lambda}^{3 \mathrm{D}}(\vec{x})$ is the eigenfunction of $i D^{3 \mathrm{D}}$ with the eigenvalue $\lambda$, and $\tau_{3} \phi_{ \pm}\left(x_{4}\right)= \pm \phi_{ \pm}\left(x_{4}\right)$ represent the \pm chiral modes. The APS boundary condition is expressed by

$$
\begin{aligned}
& \left.\phi_{+}\left(x_{4}\right)\right|_{x_{4}=0}=0,\left.\quad\left(\partial_{4}-\lambda\right) \phi_{-}\left(x_{4}\right)\right|_{x_{4}=0}=0, \quad \text { for } \lambda \geq 0, \\
& \left.\phi_{-}\left(x_{4}\right)\right|_{x_{4}=0}=0,\left.\quad\left(\partial_{4}+\lambda\right) \phi_{+}\left(x_{4}\right)\right|_{x_{4}=0}=0, \quad \text { for } \lambda<0 .
\end{aligned}
$$


Let us solve the equation Eq. (17) for the case $\lambda \geq 0$. One immediately obtains

$$
\begin{aligned}
\phi_{+}^{\omega}\left(x_{4}\right) & =\frac{u_{+}}{\sqrt{2 \pi}}\left(e^{i \omega x_{4}}-e^{-i \omega x_{4}}\right), \\
\phi_{-}^{\omega}\left(x_{4}\right) & =\frac{u_{-}}{\sqrt{2 \pi\left(\omega^{2}+\lambda^{2}\right)}}\left((i \omega+\lambda) e^{i \omega x_{4}}+(i \omega-\lambda) e^{-i \omega x_{4}}\right),
\end{aligned}
$$

where

$$
u_{+}=\left(\begin{array}{l}
1 \\
0
\end{array}\right), \quad u_{-}=\left(\begin{array}{l}
0 \\
1
\end{array}\right)
$$

and $\omega=\sqrt{\Lambda^{2}-\lambda^{2}}$. Both solutions satisfy

$$
\int_{0}^{\infty} d x_{4}\left[\phi_{ \pm}^{\omega^{\prime}}\left(x_{4}\right)\right]^{\dagger} \phi_{ \pm}^{\omega}\left(x_{4}\right)=\delta\left(\omega-\omega^{\prime}\right)
$$

for positive $\omega$ and $\omega^{\prime}$. They also satisfy in a subspace where $i D^{3 \mathrm{D}}$ takes the eigenvalue $\lambda$,

$$
\sum_{g= \pm} \int_{0}^{\infty} d \omega\left[\phi_{g}^{\omega}\left(x_{4}\right)\right]\left[\phi_{g}^{\omega}\left(x_{4}^{\prime}\right)\right]^{\dagger}=\delta\left(x_{4}-x_{4}^{\prime}\right) 1_{2 \times 2}
$$

for $x_{4}, x_{4}^{\prime}>0$. Namely, $\phi_{ \pm}^{\omega}\left(x_{4}\right)$ forms a complete set in the $x_{4}$ direction for each eigenmode of three-dimensional operator $i D^{3 \mathrm{D}}$. Note that $\Lambda^{2}>\lambda^{2}$ is always required so that no edgelocalized zero mode is allowed to exist.

Next, let us compute the kernel of the operator $\gamma_{5} e^{-t D^{\dagger} D}$ using the complete set $\phi_{ \pm}^{\omega}\left(x_{4}\right)$ obtained above for each $\lambda$. The ++ component is a simple Gaussian integral leading to

$$
\begin{aligned}
\left\langle x_{4} ;+\left|\gamma_{5} e^{-t D^{\dagger} D}\right| x_{4}^{\prime} ;+\right\rangle & =\int_{0}^{\infty} d \omega e^{-t\left(\omega^{2}+\lambda^{2}\right)}\left[\phi_{+}^{\omega}\left(x_{4}\right)\right]_{+}\left[\phi_{+}^{\omega}\left(x_{4}^{\prime}\right)\right]_{+}^{*} \\
& =\frac{e^{-\lambda^{2} t}}{\sqrt{4 \pi t}}\left[e^{-\frac{\left(x_{4}-x_{4}^{\prime}\right)^{2}}{4 t}}-e^{-\frac{\left(x_{4}+x_{4}^{\prime}\right)^{2}}{4 t}}\right],
\end{aligned}
$$

where we have used the bracket notation $\left[\phi_{ \pm}^{\omega}\left(x_{4}\right)\right]_{g}=\left\langle x_{4} ; g \mid \omega ; \pm\right\rangle$ (note here that $\left[u_{ \pm}\right]_{g}$ has nonzero component only for $g= \pm$ ). The -- component needs a little trick to evaluate,

$$
\begin{aligned}
\left\langle x_{4} ;-\left|\gamma_{5} e^{-t D^{\dagger} D}\right| x_{4}^{\prime} ;-\right\rangle & =\int_{0}^{\infty} d \omega e^{-t\left(\omega^{2}+\lambda^{2}\right)}\left[\phi_{-}^{\omega}\left(x_{4}\right)\right]_{-}\left[\phi_{-}^{\omega}\left(x_{4}^{\prime}\right)\right]_{-}^{*} \\
& =\frac{e^{-\lambda^{2} t}}{\sqrt{4 \pi t}}\left[e^{-\frac{\left(x_{4}-x_{4}^{\prime}\right)^{2}}{4 t}}+e^{-\frac{\left(x_{4}+x_{4}^{\prime}\right)^{2}}{4 t}}\right]+I\left(x_{4}+x_{4}^{\prime}\right),
\end{aligned}
$$

where

$$
\begin{aligned}
I\left(x_{4}+x_{4}^{\prime}\right) & =e^{-t \lambda^{2}} \int_{0}^{\infty} \frac{d \omega}{2 \pi} e^{-t \omega^{2}}\left[\frac{-2 i \lambda(\omega-i \lambda)}{\omega^{2}+\lambda^{2}} e^{i \omega\left(x_{4}+x_{4}^{\prime}\right)}+h . c .\right] \\
& =e^{-t \lambda^{2}} \int_{-\infty}^{\infty} \frac{d \omega}{2 \pi} e^{-t \omega^{2}}\left[\frac{-2 i \lambda}{\omega+i \lambda} e^{i \omega\left(x_{4}+x_{4}^{\prime}\right)}\right] .
\end{aligned}
$$


Note here that the integrand has a pole at $\omega=i \lambda$. In fact, this pole is the origin of the $\eta$ invariant. $I\left(x_{4}+x_{4}^{\prime}\right)$ satisfies a differential equation

$$
\left(\frac{\partial}{\partial x_{4}}-\lambda\right) I\left(x_{4}+x_{4}^{\prime}\right)=\frac{\lambda}{\sqrt{\pi t}} e^{-t \lambda^{2}} e^{-\frac{\left(x_{4}+x_{x}^{\prime}\right)^{2}}{4 t}} .
$$

Here, the solution of Eq. (27) is given by

$$
I\left(x_{4}+x_{4}^{\prime}\right)=-\lambda e^{\lambda\left(x_{4}+x_{4}^{\prime}\right)}\left\{\operatorname{erfc}\left(\frac{x_{4}+x_{4}^{\prime}}{2 \sqrt{t}}+\lambda \sqrt{t}\right)+c\right\}
$$

where the function erfc denotes the complementary error function,

$$
\operatorname{erfc}(x)=\frac{2}{\sqrt{\pi}} \int_{x}^{\infty} d \xi e^{-\xi^{2}}
$$

Since it takes $\operatorname{erfc}(-\infty)=2, \operatorname{erfc}(0)=1$, and $\operatorname{erfc}(\infty)=0$, the constant $c$ must be zero in order to satisfy the $t \rightarrow 0$ limit converging to Eq. (23).

For $\lambda<0$, we obtain the same formula but with $\lambda$ and $\phi_{ \pm}^{\omega}$ being replaced by $-\lambda$, and $\phi_{\mp}^{\omega}$. Combining these results, the kernel is evaluated as

$$
\sum_{g= \pm}\left\langle x_{4} ; g\left|\gamma_{5} e^{-t D^{\dagger} D}\right| x_{4}^{\prime} ; g\right\rangle=\operatorname{sign} \lambda\left[-\frac{e^{-\lambda^{2} t}}{\sqrt{\pi t}} e^{-\frac{\left(x_{4}+x_{4}^{\prime}\right)^{2}}{4 t}}+|\lambda| e^{|\lambda|\left(x_{4}+x_{4}^{\prime}\right)} \operatorname{erfc}\left(\frac{x_{4}+x_{4}^{\prime}}{2 \sqrt{t}}+|\lambda| \sqrt{t}\right)\right] \text {. }
$$

We can compute the index by taking a trace over $x$ and $\lambda$,

$$
\begin{aligned}
\operatorname{Tr} \gamma_{5} e^{-t D^{\dagger} D} & =\sum_{\lambda} \operatorname{sign} \lambda \int d x_{4} \frac{\partial}{\partial x_{4}}\left[\frac{1}{2} e^{2|\lambda| x_{4}} \operatorname{erfc}\left(\frac{x_{4}}{\sqrt{t}}+|\lambda| \sqrt{t}\right)\right] \int_{Y} d^{3} y\left|\phi_{\lambda}^{3 \mathrm{D}}(\vec{y})\right|^{2} \\
& =-\sum_{\lambda} \frac{\operatorname{sign} \lambda}{2} \operatorname{erfc}(|\lambda| \sqrt{t}) .
\end{aligned}
$$

Taking the $t=0$ limit, we obtain the desired formula,

$$
\lim _{t \rightarrow 0} \operatorname{Tr} \gamma_{5} e^{-t D^{\dagger} D}=-\sum_{\lambda} \frac{\operatorname{sign} \lambda}{2}=-\frac{\eta\left(i D^{3 \mathrm{D}}\right)}{2} .
$$

It is important to note again that the APS boundary condition allows no edge-localized modes. The eta-invariant appears from a non-trivial $\omega$ integration over the bulk modes, which looks very different from what we expect in physics of topological insulators. 


\section{B. Anti-APS boundary condition}

It is interesting to consider the anti-APS boundary condition for the same set-up, where $A$ has no $x_{4}$ dependence. As mentioned before, the crucial difference from the APS boundary condition is the existence of the edge-localized modes. The condition

$$
\begin{aligned}
& \left.\phi_{-}\left(x_{4}\right)\right|_{x_{4}=0}=0,\left.\quad\left(\partial_{4}+\lambda\right) \phi_{+}\left(x_{4}\right)\right|_{x_{4}=0}=0, \quad \text { for } \lambda \geq 0, \\
& \left.\phi_{+}\left(x_{4}\right)\right|_{x_{4}=0}=0,\left.\quad\left(\partial_{4}-\lambda\right) \phi_{-}\left(x_{4}\right)\right|_{x_{4}=0}=0, \quad \text { for } \lambda<0,
\end{aligned}
$$

allows the edge-localized chiral zero modes,

$$
\begin{aligned}
& \phi_{+}^{\text {edge }}\left(x_{4}\right)=u_{+} \sqrt{2 \lambda} e^{-\lambda x_{4}}, \quad \text { for } \lambda \geq 0, \\
& \phi_{-}^{\text {edge }}\left(x_{4}\right)=u_{-} \sqrt{2|\lambda|} e^{\lambda x_{4}}, \quad \text { for } \lambda<0,
\end{aligned}
$$

which satisfy $D^{\dagger} D \phi_{ \pm}^{\text {edge }}\left(x_{4}\right)=0$.

As in the previous section, let us compute the case $\lambda \geq 0$. First, we note that the edge-localized zero mode is isolated from the bulk nonzero modes,

$$
\begin{aligned}
\phi_{+}^{\omega}\left(x_{4}\right) & =\frac{u_{+}}{\sqrt{2 \pi\left(\omega^{2}+\lambda^{2}\right)}}\left((i \omega-\lambda) e^{i \omega x_{4}}+(i \omega+\lambda) e^{-i \omega x_{4}}\right), \\
\phi_{-}^{\omega}\left(x_{4}\right) & =\frac{u_{-}}{\sqrt{2 \pi}}\left(e^{i \omega x_{4}}-e^{-i \omega x_{4}}\right),
\end{aligned}
$$

where $\omega=\sqrt{\Lambda^{2}-\lambda^{2}}$ must be a real number. In fact, in contrast to the - chirality sector, the completeness in the + chirality sector is not achieved by the bulk nonzero modes $\phi_{+}^{\omega}$ alone,

$$
\int_{0}^{\infty} d \omega\left[\phi_{+}^{\omega}\left(x_{4}\right)\right]_{+}\left[\phi_{+}^{\omega}\left(x_{4}^{\prime}\right)\right]_{+}^{*}=\delta\left(x_{4}-x_{4}^{\prime}\right)-2 \lambda e^{-\lambda\left(x_{4}+x_{4}^{\prime}\right)},
$$

whose second term is only canceled by adding $\left[\phi_{+}^{\text {edge }}\left(x_{4}\right)\right]_{+}\left[\phi_{+}^{\text {edge }}\left(x_{4}^{\prime}\right)\right]_{+}^{*}$.

Next, let us compute the kernel of the operator $\gamma_{5} e^{-t D^{\dagger} D}$,

$$
\begin{aligned}
\sum_{g= \pm}\left\langle x_{4} ; g\left|\gamma_{5} e^{-t D^{\dagger} D}\right| x_{4}^{\prime} ; g\right\rangle= & \int_{0}^{\infty} d \omega e^{-t\left(\omega^{2}+\lambda^{2}\right)}\left[\phi_{+}^{\omega}\left(x_{4}\right)\right]_{+}\left[\phi_{+}^{\omega}\left(x_{4}^{\prime}\right)\right]_{+}^{*}+\left[\phi_{+}^{\text {edge }}\left(x_{4}\right)\right]_{+}\left[\phi_{+}^{\text {edge }}\left(x_{4}^{\prime}\right)\right]_{+}^{*} \\
& -\int_{0}^{\infty} d \omega e^{-t\left(\omega^{2}+\lambda^{2}\right)}\left[\phi_{-}^{\omega}\left(x_{4}\right)\right]_{-}\left[\phi_{-}^{\omega}\left(x_{4}^{\prime}\right)\right]_{-}^{*}
\end{aligned}
$$

The second and third terms are easily obtained,

$$
\begin{aligned}
\int_{0}^{\infty} d \omega e^{-t\left(\omega^{2}+\lambda^{2}\right)}\left[\phi_{-}^{\omega}\left(x_{4}\right)\right]_{-}\left[\phi_{-}^{\omega}\left(x_{4}^{\prime}\right)\right]_{-}^{*} & =\frac{e^{-\lambda^{2} t}}{\sqrt{4 \pi t}}\left[e^{-\frac{\left(x_{4}-x_{4}^{\prime}\right)^{2}}{4 t}}-e^{-\frac{\left(x_{4}+x_{4}^{\prime}\right)^{2}}{4 t}}\right], \\
{\left[\phi_{+}^{\text {edge }}\left(x_{4}\right)\right]_{+}\left[\phi_{+}^{\text {edge }}\left(x_{4}^{\prime}\right)\right]_{+}^{*} } & =2 \lambda e^{-\lambda\left(x_{4}+x_{4}^{\prime}\right)}
\end{aligned}
$$


while the first term becomes

$$
\int_{0}^{\infty} d \omega e^{-t\left(\omega^{2}+\lambda^{2}\right)}\left[\phi_{+}^{\omega}\left(x_{4}\right)\right]_{+}\left[\phi_{+}^{\omega}\left(x_{4}^{\prime}\right)\right]_{+}^{*}=\frac{e^{-\lambda^{2} t}}{\sqrt{4 \pi t}}\left[e^{-\frac{\left(x_{4}-x_{4}^{\prime}\right)^{2}}{4 t}}+e^{-\frac{\left(x_{4}+x_{4}^{\prime}\right)^{2}}{4 t}}\right]+I^{\prime}\left(x_{4}+x_{4}^{\prime}\right),
$$

where

$$
I^{\prime}\left(x_{4}+x_{4}^{\prime}\right)=-\lambda e^{-\lambda\left(x_{4}+x_{4}^{\prime}\right)} \operatorname{erfc}\left(-\frac{x_{4}+x_{4}^{\prime}}{2 \sqrt{t}}+\lambda \sqrt{t}\right) .
$$

For $\lambda<0$, we obtain the same formula but with $\lambda$ and $\phi_{ \pm}^{\omega}$ being replaced by $-\lambda$ and $\phi_{\mp}^{\omega}$. Combining these results, we obtain

$$
\begin{aligned}
\sum_{g= \pm}\left\langle x_{4} ; g\left|\gamma_{5} e^{-t D^{\dagger} D}\right| x_{4}^{\prime} ; g\right\rangle= & \operatorname{sign} \lambda\left[\frac{e^{-\lambda^{2} t}}{\sqrt{\pi t}} e^{-\frac{\left(x_{4}+x_{4}^{\prime}\right)^{2}}{4 t}}\right. \\
& \left.-|\lambda| e^{-|\lambda|\left(x_{4}+x_{4}^{\prime}\right)}\left\{\operatorname{erfc}\left(-\frac{x_{4}+x_{4}^{\prime}}{2 \sqrt{t}}+|\lambda| \sqrt{t}\right)-2\right\}\right] .
\end{aligned}
$$

Now we are ready to compute the index by taking trace over $x$ and $\lambda$,

$$
\begin{aligned}
\operatorname{Tr} \gamma_{5} e^{-t D^{\dagger} D} & =\sum_{\lambda} \operatorname{sign} \lambda \int d x_{4}\left[\frac{\partial}{\partial x_{4}}\left\{\frac{1}{2} e^{-2|\lambda| x_{4}} \operatorname{erfc}\left(-\frac{x_{4}}{\sqrt{t}}+|\lambda| \sqrt{t}\right)\right\}+2|\lambda| e^{-2|\lambda| x_{4}}\right] \\
& =-\sum_{\lambda} \frac{\operatorname{sign} \lambda}{2} \operatorname{erfc}(|\lambda| \sqrt{t})+\sum_{\lambda} \operatorname{sign} \lambda .
\end{aligned}
$$

In the $t \rightarrow 0$ limit, the above formula apparently converges to

$$
\lim _{t \rightarrow 0} \operatorname{Tr} \gamma_{5} e^{-t D^{\dagger} D}=\sum_{\lambda} \frac{\operatorname{sign} \lambda}{2}=\frac{\eta\left(i D^{3 \mathrm{D}}\right)}{2},
$$

which has the opposite sign to the APS case in Eq. (32). We should, however, note that the two terms in Eq. (45) have different origins. The first term is a contribution from the nonzero bulk modes, which is exactly the same as the APS boundary case. But the second contribution is from the edge-localized zero energy modes, which cannot be regularized by the exponential factor $e^{-t D^{\dagger} D}$. For this reason, the anti-APS boundary case is not appropriate for deriving the index theorem, since the simple heat-kernel-type regularization is not enough to regulate these edge-localized modes.

\section{General gauge background}

The APS index theorem applies only to a compact manifold. Therefore, the infinite flat cylinder computation in the previous sections is not complete, as clearly seen by the fact that 
$\eta\left(i D^{3 \mathrm{D}}\right) / 2$ alone cannot be an integer in general. On a compact manifold, the "flatness" in the $x_{4}$ direction must be lost to make the system compactified, otherwise, we need another boundary, which cancels (the noninteger part of) the eta invariant. The original APS index theorem [5] was completed by introducing "doubling" of a non-flat compact manifold $X$ to eliminate the boundary and form a closed manifold, and then interpolating the solutions of flat cylinder and those on the doubled $X$. Here they still assumed a flatness near the boundary, so that the flat cylinder solutions well approximate the full ones.

Let us here review the derivation by Alvarez-Gaumé et al. [15] who introduced two boundaries at $t=-\infty$ and $+\infty$, so that the flat metric is allowed, and consider a non-trivial $x_{4}$ dependence of the gauge fields (here we take $U(1)$ or $S U(N)$ gauge group) between them to derive the index.

First, the Dirac operator is expressed as

$$
D=\tau_{1} \otimes 1_{2 \times 2} \frac{\partial}{\partial t}-i \tau_{2} \otimes H_{t}
$$

where $\tau_{i}$ denote the Pauli matrices, and $H_{t}=i D^{3 \mathrm{D}}\left(x_{4}=t\right)$. In the adiabatic approximation, where $H_{t}$ changes slowly with $t$, the zero mode solution of $D$ is given by

$$
\begin{aligned}
D \psi & =0, \quad \psi=f(t) \otimes \psi_{t} \\
H_{t} \psi_{t} & =\lambda(t) \psi_{t}, \quad \tau_{1}\left(\partial_{t}+\tau_{3} \lambda(t)\right) f(t)=0 \\
f(t) & =\exp \left(-\int^{t} d t^{\prime} \tau_{3} \lambda\left(t^{\prime}\right)\right) \chi
\end{aligned}
$$

where $\chi$ is a constant. For the positive chiral mode (here $\left.\tau_{3}=+1\right) f(t)$ is normalizable only when $\lambda(-\infty)<0$ and $\lambda(+\infty)>0$, while the negative chiral mode has opposite signs of the eigenvalue. In either case, $\lambda(t)$ changes its sign somewhere in the $t$ history. Namely, the APS index counts the zero-crossings of the eigenvalues of $H_{t}$, which can be expressed by

$$
\mathcal{I}=\frac{1}{2}\left[\eta\left(H_{t=+\infty}\right)-\eta\left(H_{t=-\infty}\right)\right]-\frac{1}{2} \int_{-\infty}^{\infty} d t^{\prime} \frac{d}{d t^{\prime}} \eta\left(H_{t^{\prime}}\right),
$$

where the second term is necessary to cancel the non-integer part of the eta invariants.

The remaining task is to show that the second term is equivalent to the four-dimensional integral of the conventional instanton density. To this end, we first express the eta-invariant in integral-form

$$
\eta\left(H_{t}\right)=\lim _{s \rightarrow 0} \frac{2}{\Gamma\left(\frac{s+1}{2}\right)} \int_{0}^{\infty} d u u^{s} \operatorname{Tr} H_{t} e^{-u^{2} H_{t}^{2}}
$$


and compute its $t$ derivative,

$$
\begin{aligned}
-\frac{1}{2} \frac{d}{d t} \eta\left(H_{t}\right) & =-\lim _{s \rightarrow 0} \frac{1}{\Gamma\left(\frac{s+1}{2}\right)} \int_{0}^{\infty} d u u^{s} \frac{\partial}{\partial u} \operatorname{Tr}\left[u \frac{\partial H_{t}}{\partial t} e^{-u^{2} H_{t}^{2}}\right] \\
& =\frac{1}{\sqrt{\pi}} \lim _{u \rightarrow 0} \operatorname{Tr}\left[u \frac{\partial H_{t}}{\partial t} e^{-u^{2} H_{t}^{2}}\right]
\end{aligned}
$$

where we have taken the $s \rightarrow 0$ limit and the trace $\operatorname{Tr}$ is taken over two-component spinor, color, and three-dimensional coordinates. Then one can relate this quantity to the threedimensional integral of the instanton density at $x_{4}=t$ by

$$
\begin{aligned}
\int d^{3} x \epsilon_{\mu \nu \rho \sigma} \operatorname{tr}_{c} F^{\mu \nu} F^{\rho \sigma}\left(x_{4}=t\right) & =\lim _{u \rightarrow 0} \int d^{3} x \operatorname{tr}_{c, s} \gamma_{5} e^{u^{2} D^{2}} \\
& =\lim _{u \rightarrow 0} \operatorname{Tr}_{\operatorname{tr}_{s^{\prime}}} \tau_{3} e^{u^{2}\left(\partial_{t}^{2}-H_{t}^{2}+\tau_{3} \frac{\partial H_{t}}{\partial t}\right)} \\
& =\lim _{u \rightarrow 0} \int_{-\infty}^{\infty} \frac{d \omega}{2 \pi} \operatorname{Tr}_{\operatorname{tr}_{s^{\prime}}} \tau_{3} e^{u^{2}\left(\left(i \omega+\partial_{t}\right)^{2}-H_{t}^{2}+\tau_{3} \frac{\partial H_{t}}{\partial t}\right)} \\
& =\lim _{u \rightarrow 0} \int_{-\infty}^{\infty} \frac{d \omega}{2 \pi} e^{-\omega^{2} u^{2}} \operatorname{Tr}_{\operatorname{tr}_{s^{\prime}}} \tau_{3}\left(u^{2} \tau_{3} \frac{\partial H_{t}}{\partial t}\right) e^{-u^{2} H_{t}^{2}} \\
& =\frac{1}{\sqrt{\pi}} \lim _{u \rightarrow 0} \operatorname{Tr}\left[u \frac{\partial H_{t}}{\partial t} e^{-u^{2} H_{t}^{2}}\right]
\end{aligned}
$$

which agrees with Eq. (53). Identifying $t=x_{4}$ and $H_{t}=-i D^{3 \mathrm{D}}\left(x_{4}=t\right)$, we obtain

$$
\mathcal{I}=\int d^{4} x \epsilon_{\mu \nu \rho \sigma} \operatorname{tr}_{c} F^{\mu \nu} F^{\rho \sigma}-\frac{1}{2}\left[\eta\left(i D^{3 \mathrm{D}}(-\infty)\right)-\eta\left(i D^{3 \mathrm{D}}(+\infty)\right)\right]
$$

It is important to note that the above result is obtained by the standard Fujikawa method: inserting the conventional plane wave solutions in the $x_{4}$ direction. This is valid only when $t$ dependence is negligible at the boundaries $t= \pm \infty$. Namely, this computation is done in a set-up where the role of edge modes is not relevant. The interactions between edge and bulk modes are turned off. For the more general $x_{4}$ dependent gauge background, the standard Fujikawa method is difficult since the APS boundary condition requires non-perturbative information of the eigenfunctions of $D^{3 \mathrm{D}}$. As is discussed later, the APS boundary condition has more fundamental problems in application to the physical fermion system with boundaries.

\section{Difference between the $\eta$ invariant and the Chern-Simons term}

So far, the $\eta$ invariant has been defined by the eigenvalues of the Dirac operator on the surface, and it has not been shown how it is perturbatively expressed. It is known that 
$\eta\left(i D^{3 \mathrm{D}}\right)$ formally appears in the phase of a "massive" Dirac fermion determinant,

$$
\operatorname{det} \frac{D^{3 \mathrm{D}}-M}{D^{3 \mathrm{D}}+\Lambda} \propto \exp \left[i \pi \eta\left(i D^{3 \mathrm{D}}\right)\right]
$$

where we have introduced the Pauli-Villars regulator, assuming both $M$ and $\Lambda$ are positive (and large), and it is perturbatively equivalent to

$$
\exp (i C S)
$$

which can be obtained from an integral

$$
C S=\int_{0}^{u} d u \frac{d}{d u} \operatorname{Im} \ln \operatorname{det} \frac{D^{3 \mathrm{D}}(u)-M}{D^{3 \mathrm{D}}(u)+\Lambda},
$$

up to $1 / M$ and $1 / \Lambda$ corrections. Here $D^{3 \mathrm{D}}(u)$ is the Dirac operator with $u A_{\mu}$, which denotes a linear one-parameter deformation of the original gauge field.

In the above massive fermion determinant, it is no problem to identify the Chern-Simons action $C S$ with the $\eta$ invariant. However, in the index theorem, they are different, since $C S$ is not gauge invariant under a "large" gauge transformation with a winding number $n$,

$$
C S \rightarrow C S+2 \pi n
$$

Since $\eta\left(i D^{3 \mathrm{D}}\right) / 2$ should be obtained in a gauge invariant regularization, it differs from $C S / 2 \pi$ by an integer, which is not gauge invariant.

In Appendix A, we exactly compute the $\eta$ invariant in one-dimensional QED with flat background field and obtain

$$
\frac{\eta}{2}=\frac{C S}{2 \pi}-\left[\frac{C S}{2 \pi}\right]
$$

(up to an irrelevant constant) where $[f]$ denotes the Gauss symbol or the greatest integer less than or equal to $f$. Although we have not found any proof in the literature, we assume that this expression is generally valid in the following discussions ${ }^{2}$, even for the three-dimensional case with non-Abelian gauge fields. In fact, Eq. (60) has good properties listed below. It is 1) manifestly gauge invariant, 2) reflects non-locality of the APS boundary condition as the Gauss symbol is highly nonlocal, 3) shows that the total APS index is no longer

2 Our argument cannot exclude a possibility of an additional gauge invariant integer, which is non-locally given. 
a topological invariant, since $\eta / 2$ can discretely jump by an integer ${ }^{3}$, and 4 ) shows noncompatibility of the gauge invariance and the $\mathrm{T}$ (or parity) invariance of the massless Dirac fermion determinant. To confirm the last property, let us consider the massless fermion determinant with Pauli-Villars regulator,

$$
\operatorname{det} \frac{D^{3 \mathrm{D}}}{D^{3 \mathrm{D}}+\Lambda} \propto \exp \left[i \pi \eta\left(i D^{3 \mathrm{D}}\right) / 2\right]
$$

which is gauge invariant but breaks the $\mathrm{T}$ invariance. To recover the $\mathrm{T}$ invariance, the only possible local counterterm we can add is $\exp (-i C S / 2)$; then the remaining phase $\exp (-i \pi[C S / 2 \pi])$ breaks the gauge invariance [13, by the same mechanism as Witten's global anomaly [34].

\section{E. APS boundary condition unlikely to be realized in physics}

The APS boundary condition commutes with $\gamma_{5}$, and, therefore, preserves helicity. Namely this boundary condition keeps the fermion, on which the Dirac operator operates, massless [35]. This looks like a reasonable choice but when we consider reflection of the fermions at the boundary, we find that the APS boundary condition is very unnatural ${ }^{4}$.

Consider a flat surface of some material at $x_{4}=0$. Unless the boundary fermion is somehow polarized, for example, by an anisotropic crystal structure, it is natural to assume that the system is rotationally symmetric along the $x_{4}$ axis perpendicular to the surface. This $S O(3)$ (or $S O(2,1)$ in Minkowski space-time) rotational symmetry is essential for the edge-localized mode of topological insulators to act as a relativistic Dirac fermion. However, this $S O(3)$ symmetry is not compatible with the helicity conservation, which is respected by the APS boundary condition, because it requires a spin flip whenever fermions are reflected at the boundary [36].

In mathematics, we can impose any boundary condition on the first order differential equations. However, this is not true in quantum field theory, where we need to regularize them by subtraction equations and take the continuum limit. In the lattice gauge theory, it is known that any local boundary condition except for the Dirichlet boundary requires a

\footnotetext{
3 This jump is induced by the level crossing of the surface Dirac operator.

4 One may consider a possibility that fermions are never reflected, which is another unnatural set-up, where energy that fermion carries is accumulated at the boundary and never goes back to the bulk.
} 
fine tuning or some additional symmetry on the boundary to protect it in the continuum limit. As the quantum field theory is formulated to somehow neglect short-range structure, this requirement of fine-tuning should be universal for any regularization. Since the APS boundary condition is nonlocal, this argument cannot be directly applied, but it is unlikely that the nonlocality helps to make the boundary condition stable. Therefore, we conclude that the APS boundary condition is unlikely to be realized in the physical fermion system with boundary.

Treating a manifold with boundary as a closed system is also unnatural in physics, as any boundary in our world has "outside" of it. The surface of the topological insulator is nontrivial because its outside is not empty but surrounded by normal insulator.

The above discussion suggests to us a need to consider more natural set-up in physics. We should have a domain-wall, like the one between topological and normal insulators, rather than a simple boundary without an outside. It is more natural to have a massive fermion since it is not the helicity but rotational symmetry that should be preserved. It is better to have a boundary condition not imposed by hand but automatically and locally given by dynamics of the system.

Can we still define an index for such a massive Dirac operator? As is shown in the next section, the answer is "yes". We introduce the so-called domain-wall Dirac fermion operator, which is massive in the bulk and gapless at the boundary. Its local boundary condition is not imposed by hand but automatically satisfied by the kink structure of the mass term. Therefore, no fine-tuning is needed. We define an index by its eta-invariant, to which the edge-localized gapless modes play a crucial role. Moreover, the new index coincides with the original APS index.

\section{APS INDEX FROM DOMAIN-WALL FERMION DIRAC OPERATOR}

In this section, we consider a different setup from the original work by APS [5]. So far we have considered a manifold with boundary, as a closed system. But in real physics, no boundary can exist without "outside" of the region. For example, the boundary of the topological insulator is always surrounded by the normal insulator. We cannot say on which the edge-localized modes reside, since they require both sides to support them, unless the gap is infinitely large. 
In this respect, the so-called domain-wall fermion [30, 31] is a more appropriate setup for the physical system with boundary. The domain-wall fermion Dirac operator is defined by

$$
D_{D W}=D+M \epsilon\left(x_{4}\right), \quad \epsilon\left(x_{4}\right)=\operatorname{sign} x_{4} .
$$

where the mass term flips its sign across the domain-wall located at $x_{4}=0$. Here and in the following, we take $M$ to be positive. In lattice gauge theory, we often consider the domain-wall fermion determinant together with a Pauli-Villars field,

$$
\operatorname{det} \frac{D+M \epsilon\left(x_{4}\right)}{D-M},
$$

to cancel the bulk mode effects in the region $x_{4}<0$. Note here that fermion field is defined in the whole $-\infty<x_{4}<\infty$ region and no boundary condition is imposed on it ${ }^{5}$. Therefore, this determinant provides a good model to describe fermions in a topological insulator located in the $x_{4}>0$ region, surrounded by a normal insulator sitting in the $x_{4}<0$ region. As we explicitly show, the edge-localized modes appear at the boundary $x_{4}=0$, and play a crucial role in the definition of the index.

The determinant Eq. (63) is real ${ }^{6}$, due to the " $\gamma_{5}$ Hermiticity",

$$
\begin{aligned}
\operatorname{det}\left[\left(D+M \epsilon\left(x_{4}\right)\right)(D-M)^{-1}\right] & =\operatorname{det}\left[\gamma_{5}^{2}\left(D+M \epsilon\left(x_{4}\right)\right) \gamma_{5}^{2}(D-M)^{-1}\right] \\
& =\operatorname{det}\left[\left(D^{\dagger}+M \epsilon\left(x_{4}\right)\right)\left(D^{\dagger}-M\right)^{-1}\right] \\
& =\left|\operatorname{det}\left[\left(D^{\dagger}+M \epsilon\left(x_{4}\right)\right)\left(D^{\dagger}-M\right)^{-1}\right]\right|(-1)^{\mathcal{I}},
\end{aligned}
$$

where $\mathcal{I}$ is an integer determining the sign of the determinant. In fact, we will explicitly show that this integer $\mathcal{I}$ is equivalent to the APS index. A similar statement is found in [17], but neither the explicit bulk fermion determinant nor its boundary condition is given. The outside of our target domain is not mentioned, either. As is shown below, we need neither the massless Dirac operator nor nonlocal APS boundary condition for the new index.

Our new index $\mathcal{I}$ is formally defined by a regularized eta invariant of the Hermitian operator $H_{D W}=\gamma_{5}\left(D+M \epsilon\left(x_{4}\right)\right)$ :

$$
\mathcal{I} \equiv \frac{\eta\left(H_{D W}^{r e g}\right)}{2}=\frac{1}{2} \eta\left(H_{D W}\right)-\frac{1}{2} \eta\left(H_{P V}\right)
$$

5 Strictly speaking, we should give an IR cutoff by compactifying the manifold with some appropriate boundary condition, such as periodic boundary condition. Then we need another domain-wall at some point of $x_{4}$. We will discuss this anti-domain-wall fermion contribution at the end of this section.

6 This is true even with a naive lattice regularization using the Wilson Dirac operator. 
where we employ the Pauli-Villars regularization with another Hermitian operator $H_{P V}=$ $\gamma_{5}(D-M)$. This definition coincides with the exponent appearing in Eq. (64) as

$$
\begin{aligned}
\operatorname{det} \frac{D+M \epsilon\left(x_{4}\right)}{D-M} & =\operatorname{det} \frac{i H_{D W}}{i H_{P V}}=\prod_{\lambda_{D W}} i \lambda_{D W} / \prod_{\lambda_{P V}} i \lambda_{P V} \\
& \propto \exp \left(\frac{i \pi}{2}\left(\sum_{\lambda_{D W}} \operatorname{sign} \lambda_{D W}-\sum_{\lambda_{P V}} \operatorname{sign} \lambda_{P V}\right)\right) \\
& =(-1)^{\frac{1}{2} \eta\left(H_{D W}\right)-\frac{1}{2} \eta\left(H_{P V}\right)} .
\end{aligned}
$$

In the following, we compute the two eta invariants $\eta\left(H_{D W}\right)$ and $\eta\left(H_{P V}\right)$ separately, by introducing another regularization using the (generalized) $\zeta$ function (we simply call it the $\zeta$-function regularization). This double regularization is not theoretically needed but simplifies the computation and clarifies the role of the Pauli-Villars fields. In fact, we see that $\eta\left(H_{D W}\right) / 2$ alone gives only a "half" of the (bulk contribution of) total APS index, to which another "half" is provided by $\eta\left(H_{P V}\right) / 2$.

Let us compute $\eta\left(H_{P V}\right)$ first. Interestingly, it coincides with the AS index,

$$
\begin{aligned}
\eta\left(H_{P V}\right) & =\lim _{s \rightarrow 0} \operatorname{Tr} \frac{H_{P V}}{\left(\sqrt{H_{P V}^{2}}\right)^{1+s}}=\lim _{s \rightarrow 0} \frac{1}{\Gamma\left(\frac{1+s}{2}\right)} \int_{0}^{\infty} d t t^{\frac{s-1}{2}} \operatorname{Tr} H_{P V} e^{-t H_{P V}^{2}} \\
& =-\frac{1}{\sqrt{\pi}} \int_{0}^{\infty} d t^{\prime} t^{\prime-\frac{1}{2}} \operatorname{Tr} \gamma_{5}\left(1-\frac{D}{M}\right) e^{-t^{\prime} D^{\dagger} D / M^{2}} e^{-t^{\prime}}, \\
& =-\frac{1}{32 \pi^{2}} \int d^{4} x \epsilon_{\mu \nu \rho \sigma} \operatorname{tr}_{c} F^{\mu \nu} F^{\rho \sigma}+\mathcal{O}\left(1 / M^{2}\right) .
\end{aligned}
$$

Here we have changed the valuable $t=t^{\prime} / M^{2}$, and the conventional Fujikawa method has been applied to evaluate $\operatorname{Tr} \gamma_{5} e^{-t^{\prime} D^{\dagger} D / M^{2}}$. Moreover, we can show that $\eta\left(H_{P V}\right)$ is independent of $M$ as follows. Since $\left\{H_{P V}, D\right\}=0$, every eigenmode $\phi_{\lambda_{P V}}$ with eigenvalue $\lambda_{P V}$ makes a pair with $D \phi_{\lambda}$ whose eigenvalue has the opposite sign $-\lambda_{P V}$, unless $D \phi_{\lambda}=0$. The zero modes of $D$, which commute with $\gamma_{5}$, are simultaneously the eigenmodes of $H_{P V}$, whose eigenvalues are $\pm M$ with $\gamma_{5}=\mp 1$. Therefore, the left-hand side of Eq. (67) becomes

$$
\eta\left(H_{P V}\right)=-\operatorname{Tr}_{z e r o s} \frac{\gamma_{5} M}{M}=-\left(n_{+}-n_{-}\right),
$$

which is independent of $M$. Here $\operatorname{Tr}_{\text {zeros }}$ is the trace over the zero modes of $D$ only.

It is also interesting to note that the structure of the eta invariant is naturally embedded in the index theorem of the massless lattice Dirac operator. The Neuberger's lattice overlap Dirac operator [37, 38] with the lattice spacing $a$ is defined by

$$
D^{o v}=\frac{1}{a}\left[1+\gamma_{5} \frac{H_{W}}{\sqrt{H_{W}^{2}}}\right],
$$


where $H_{W}=\gamma_{5}\left(D_{W}-1 / a\right)$ is the Hermitian Wilson Dirac operator with the cut-off scale mass $M=-1 / a$. Actually, its index is given by

$$
\operatorname{Tr} \gamma_{5}\left(1-\frac{D_{o v} a}{2}\right)=-\frac{1}{2} \operatorname{Tr} \frac{H_{W}}{\sqrt{H_{W}^{2}}}=-\frac{1}{2} \eta\left(H_{W}\right),
$$

where we have used $\operatorname{Tr} \gamma_{5}=0$ with the finite cut-off. The sign is not important here: it is just a convention of the sign for the mass compared to the Wilson term, but the factor $1 / 2$ has a crucial role to cancel the contribution from the doublers, which plays the same role of (another) Pauli-Villars field in the continuum. This computation clearly shows that there is no need to introduce massless Dirac operator to define the index, at least, on a closed manifold. As we will show below, this is true even with boundary.

Now our goal in this section is to compute the remaining contribution $\eta\left(H_{D W}\right)$ and show

$$
\eta\left(H_{D W}\right)=\frac{1}{32 \pi^{2}} \int d^{4} x \epsilon\left(x_{4}\right) \epsilon_{\mu \nu \rho \sigma} \operatorname{tr}_{c} F^{\mu \nu} F^{\rho \sigma}-\eta\left(i D^{3 \mathrm{D}}\right) .
$$

For this massive case, we switch to the Dirac representation for the gamma matrices:

$$
\begin{gathered}
\gamma_{i=1,2,3}=\left(\begin{array}{c}
\sigma_{i} \\
\sigma_{i}
\end{array}\right)=\tau_{1} \otimes \sigma_{i}, \quad \gamma_{4}=\left(\begin{array}{ll}
1_{2 \times 2} & \\
-1_{2 \times 2}
\end{array}\right)=\tau_{3} \otimes 1_{2 \times 2}, \\
\gamma_{5}=-\gamma_{1} \gamma_{2} \gamma_{3} \gamma_{4}=\left(\begin{array}{cc}
i 1_{2 \times 2} \\
-i 1_{2 \times 2}
\end{array}\right)=-\tau_{2} \otimes 1_{2 \times 2} .
\end{gathered}
$$

Our Hermitian Dirac operator is then expressed by

$$
\begin{aligned}
H_{D W} & =\gamma_{5} \gamma_{4}\left(\partial_{4}+\gamma_{4} M \epsilon\left(x_{4}\right)\right)+B \\
& =\left(\begin{array}{rr}
-i\left(\partial_{4}-M \epsilon\left(x_{4}\right)\right) \\
-i\left(\partial_{4}+M \epsilon\left(x_{4}\right)\right)
\end{array}\right)+\left(\begin{array}{rr}
-i D^{3 \mathrm{D}} & \\
& i D^{3 \mathrm{D}}
\end{array}\right),
\end{aligned}
$$

where $B=\gamma_{5} \sum_{i=1}^{3} \gamma_{i} D^{i}$, and $D^{3 \mathrm{D}}=-\sigma_{i} D^{i}$.

\section{A. $x_{4}$-independent background}

As we have demonstrated in the case of the APS boundary, let us begin with the flat

background with no $x_{4}$ dependence of the gauge fields. Our cylinder is now extended to the $x_{4}<0$ region. Since $F_{4 k}=0$ for any $k$, our goal here is to show

$$
\eta\left(H_{D W}\right)=-\eta\left(i D^{3 \mathrm{D}}\right)
$$


With the $A_{4}=0$ gauge, $H_{D W}^{2}$ can be written as

$$
H_{D W}^{2}=-\partial_{4}^{2}+B^{2}+M^{2}-2 M \gamma_{4} \delta\left(x_{4}\right),
$$

which commutes with $\gamma_{4}$, and $B$. It is, therefore, convenient to consider the eigenvalue problem of $H_{D W}^{2}$ by assuming the form of the solution as $\varphi_{ \pm}\left(x_{4}\right) \otimes \phi_{\lambda}^{3 \mathrm{D}}(\vec{x})$ where $\varphi_{ \pm}\left(x_{4}\right)$ satisfies

$$
\left(-\partial_{4}^{2}+\lambda^{2}+M^{2} \mp 2 M \delta\left(x_{4}\right)\right) \varphi_{ \pm}\left(x_{4}\right)=\Lambda^{2} \varphi_{ \pm}\left(x_{4}\right),
$$

$\phi_{\lambda}^{3 \mathrm{D}}(\vec{x})$ is the eigenfunction of $i D^{3 \mathrm{D}}$ with the eigenvalue $\lambda$, and $\tau_{3} \varphi_{ \pm}\left(x_{4}\right)= \pm \varphi_{ \pm}\left(x_{4}\right)$. Note that the eigenvalue of $\tau_{3}$ corresponds to that of $\gamma_{4}$.

The solutions to Eq. (76) are obtained as

$$
\begin{aligned}
\varphi_{ \pm, o}^{\omega}\left(x_{4}\right) & =\frac{u_{ \pm}}{\sqrt{4 \pi}}\left(e^{i \omega x_{4}}-e^{-i \omega x_{4}}\right), \\
\varphi_{ \pm, e}^{\omega}\left(x_{4}\right) & =\frac{u_{ \pm}}{\sqrt{4 \pi\left(\omega^{2}+M^{2}\right)}}\left\{(i \omega \mp M) e^{i \omega\left|x_{4}\right|}+(i \omega \pm M) e^{-i \omega\left|x_{4}\right|}\right\} \\
\varphi_{+, e}^{\text {edge }}\left(x_{4}\right) & =u_{+} \sqrt{M} e^{-M\left|x_{4}\right|}
\end{aligned}
$$

where $\omega=\sqrt{\Lambda^{2}-\lambda^{2}-M^{2}}$, and the subscripts $e, o$ denote even and odd components under the time reversal $T: x_{4} \leftrightarrow-x_{4}$.

We emphasize here that we have not imposed any boundary condition by hand, but the delta-function potential automatically chooses non-trivial boundary conditions on the fermion fields:

$$
\left.\left[\frac{\partial}{\partial x_{4}} \pm M \epsilon\left(x_{4}\right)\right] \varphi_{ \pm, e}^{\omega, \text { edge }}\left(x_{4}\right)\right|_{x_{4}=0}=0, \quad \varphi_{ \pm, o}^{\omega}\left(x_{4}=0\right)=0 .
$$

More importantly, these boundary conditions respect the $S O(3)$ rotational symmetry on the $x_{4}=0$ surface, rather than helicity.

The above solutions satisfy

$$
\begin{aligned}
\int_{-\infty}^{\infty} d x_{4}\left[\varphi_{ \pm, e / o}^{\omega^{\prime}}\left(x_{4}\right)\right]^{\dagger} \varphi_{ \pm, e / o}^{\omega}\left(x_{4}\right) & =\delta\left(\omega-\omega^{\prime}\right), \\
\int_{-\infty}^{\infty} d x_{4}\left[\varphi_{+, e}^{\text {edge }}\left(x_{4}\right)\right]^{\dagger} \varphi_{+, e}^{\text {edge }}\left(x_{4}\right) & =1
\end{aligned}
$$

for positive $\omega$ and $\omega^{\prime}$. They also satisfy the completeness condition in a subspace where $i D^{3 D}$ takes the eigenvalue $\lambda$, for which $B$ takes the eigenvalue $\mp \lambda$ for $\gamma_{4}= \pm 1$ eigenmodes,

$$
\begin{aligned}
\sum_{a=e, o} \int_{0}^{\infty} d \omega\left[\varphi_{+, a}^{\omega}\left(x_{4}\right)\right]\left[\varphi_{+, a}^{\omega}\left(x_{4}^{\prime}\right)\right]^{\dagger}+\left[\varphi_{+, e}^{\text {edge }}\left(x_{4}\right)\right]\left[\varphi_{+, e}^{\text {edge }}\left(x_{4}^{\prime}\right)\right]^{\dagger} & =\delta\left(x_{4}-x_{4}^{\prime}\right) 1_{2 \times 2}, \\
\int_{0}^{\infty} d \omega\left[\varphi_{-, a}^{\omega}\left(x_{4}\right)\right]\left[\varphi_{-, a}^{\omega}\left(x_{4}^{\prime}\right)\right]^{\dagger} & =\delta\left(x_{4}-x_{4}^{\prime}\right) 1_{2 \times 2},
\end{aligned}
$$


for any $x_{4}, x_{4}^{\prime}$.

Next, let us compute the kernel of the operator $H_{D W} e^{-t H_{D W}^{2}}$ using the complete set obtained above for each $\lambda$. For the ++ component, we have

$$
\begin{aligned}
\left\langle x_{4} ;+\left|H_{D W} e^{-t H_{D W}^{2}}\right| x_{4}^{\prime} ;+\right\rangle= & -\lambda\left\{\sum_{a=e, o} \int_{0}^{\infty} d \omega e^{-t\left(\omega^{2}+\lambda^{2}+M^{2}\right)}\left[\varphi_{+, a}^{\omega}\left(x_{4}\right)\right]_{+}\left[\varphi_{+, a}^{\omega}\left(x_{4}^{\prime}\right)\right]_{+}^{*}\right. \\
& \left.+e^{-\lambda^{2} t}\left[\varphi_{+, e}^{\text {edge }}\left(x_{4}\right)\right]_{+}\left[\varphi_{+, e}^{\text {edge }}\left(x_{4}^{\prime}\right)\right]_{+}^{*}\right\} \\
= & -\lambda \frac{e^{-\left(\lambda^{2}+M^{2}\right) t}}{\sqrt{4 \pi t}} e^{-\frac{\left(x_{4}-x_{4}^{\prime}\right)^{2}}{4 t}} \\
& +\frac{\lambda M}{2} e^{-\lambda^{2} t} e^{-M\left(\left|x_{4}\right|+\left|x_{4}^{\prime}\right|\right)} \operatorname{erfc}\left(-\frac{\left|x_{4}\right|+\left|x_{4}^{\prime}\right|}{2 \sqrt{t}}+M \sqrt{t}\right) \\
& -\lambda M e^{-\lambda^{2} t} e^{-M\left(\left|x_{4}\right|+\left|x_{4}^{\prime}\right|\right)},
\end{aligned}
$$

where we have used $f\left(\left(\left|x_{4}\right|+\left|x_{4}^{\prime}\right|\right)^{2}\right)+f\left(\left(\left|x_{4}\right|-\left|x_{4}^{\prime}\right|\right)^{2}\right)=f\left(\left(x_{4}+x_{4}^{\prime}\right)^{2}\right)+f\left(\left(x_{4}-x_{4}^{\prime}\right)^{2}\right)$ for any function $f(x)$. Note that the third term is the contribution from the edge-mode. The -- component is similarly obtained as

$$
\begin{aligned}
\left\langle x_{4} ;-\left|H_{D W} e^{-t H_{D W}^{2}}\right| x_{4}^{\prime} ;-\right\rangle= & \lambda \frac{e^{-\left(\lambda^{2}+M^{2}\right) t}}{\sqrt{4 \pi t}} e^{-\frac{\left(x_{4}-x_{4}^{\prime}\right)^{2}}{4 t}} \\
& -\frac{\lambda M}{2} e^{-\lambda^{2} t} e^{M\left(\left|x_{4}\right|+\left|x_{4}^{\prime}\right|\right)} \operatorname{erfc}\left(\frac{\left|x_{4}\right|+\left|x_{4}^{\prime}\right|}{2 \sqrt{t}}+M \sqrt{t}\right) .
\end{aligned}
$$

The trace is given by

$$
\begin{aligned}
\operatorname{Tr} H_{D W} e^{-t H_{D W}^{2}}= & \frac{1}{2} \sum_{\lambda} \lambda e^{-\lambda^{2} t} \int_{-\infty}^{\infty} d x_{4}\left[M e^{-2 M\left|x_{4}\right|}\left\{\operatorname{erfc}\left(-\frac{\left|x_{4}\right|}{\sqrt{t}}+M \sqrt{t}\right)-2\right\}\right] \\
& -\frac{1}{2} \sum_{\lambda} \lambda e^{-\lambda^{2} t} \int_{-\infty}^{\infty} d x_{4}\left[M e^{2 M\left|x_{4}\right|} \operatorname{erfc}\left(\frac{\left|x_{4}\right|}{\sqrt{t}}+M \sqrt{t}\right)\right] \\
= & \frac{1}{2} \sum_{\lambda} \lambda e^{-\lambda^{2} t}[2 \operatorname{erfc}(M \sqrt{t})-2] .
\end{aligned}
$$

The first term in the parenthesis with the complementary error function is from the bulk mode, while the second term is from the edge mode.

In the $M \rightarrow \infty$ limit, $\operatorname{erfc}(M \sqrt{t})$ vanishes and we obtain the desired result,

$$
\eta\left(H_{D W}\right)=-\sum_{\lambda} \frac{\lambda}{\sqrt{\pi}} \int_{0}^{\infty} d t t^{-1 / 2} e^{-\lambda^{2} t}=-\sum_{\lambda} \frac{\lambda}{|\lambda|}=-\eta\left(i D^{3 \mathrm{D}}\right) .
$$

It is clear that the $\eta\left(i D^{3 \mathrm{D}}\right)$ comes entirely from the edge-localized modes. 


\section{B. Fujikawa method for general background}

Let us consider the general gauge field background and complete the APS index theorem using the Fujikawa method.

Here we can keep the $A_{4}=0$ gauge, and therefore, the wave functions in the $x_{4}$ direction

in the previous subsection $\varphi_{ \pm, e / o}^{\omega}$ and $\varphi_{+, e}^{\text {edge }}$ are still useful. Thus, we only need to replace the three-dimensional part of the wave function, which was given by the eigenfunction $\phi_{\lambda}^{3 \mathrm{D}}$ of $i D^{3 \mathrm{D}}$, by that of the plane wave,

$$
\phi_{\boldsymbol{p}, \uparrow \downarrow}^{3 \mathrm{D}}(\boldsymbol{x})=\frac{v_{\uparrow \downarrow}}{(2 \pi)^{3 / 2}} e^{i \boldsymbol{p} \cdot \boldsymbol{x}},
$$

where $\boldsymbol{p}=\left(p_{1}, p_{2}, p_{3}\right)$ and $\boldsymbol{x}=\left(x_{1}, x_{2}, x_{3}\right)$ are the spatial components of momentum and position, respectively ${ }^{7}$. The spin degrees of freedom are described by

$$
v_{\uparrow}=\left(\begin{array}{l}
1 \\
0
\end{array}\right), \quad v_{\downarrow}=\left(\begin{array}{l}
0 \\
1
\end{array}\right) .
$$

Let us here summarize what we will compute in this subsection. Our goal is to obtain the index for general gauge background, defined by the eta invariant,

$$
\eta\left(H_{D W}\right)=\lim _{s \rightarrow 0}\left[\operatorname{Tr}\left(M \gamma_{5} \epsilon\left(x_{4}\right)\right)\left(\sqrt{H_{D W}^{2}}\right)^{-1-s}+\operatorname{Tr}\left(\gamma_{5} D\right)\left(\sqrt{H_{D W}^{2}}\right)^{-1-s}\right] .
$$

Since the second term includes contribution from massless edge-localized modes, it is nonlocal in general. Following the general strategy to compute the "local" part of the phase of the odd-dimensional massless fermion determinant [15], we consider a one-parameter family of gauge fields $u A_{\mu}$, and take a $u$-derivative and integrate it again,

$$
\begin{array}{r}
\int_{0}^{1} d u \frac{d}{d u}\left[\operatorname{Tr}\left(H_{D W}(u)-M \gamma_{5} \epsilon\left(x_{4}\right)\right)\left(\sqrt{H_{D W}(u)^{2}}\right)^{-1-s}\right] \\
=\int_{0}^{1} d u \operatorname{Tr}\left[-s \frac{d}{d u} H_{D W}(u)\left(\sqrt{H_{D W}(u)^{2}}\right)^{-1-s}-\frac{d}{d u}\left(\gamma_{5} M \epsilon\left(x_{4}\right)\left(\sqrt{H_{D W}(u)^{2}}\right)^{-1-s}\right)\right]
\end{array}
$$

where $H_{D W}(u)$ is the corresponding domain-wall fermion Dirac operator at $u$. This procedure allows us to compute the eta invariant up to an integer, which may depend on a winding number of gauge transformation on the surface. Using the formula

$$
\frac{1}{\left(\sqrt{O^{2}}\right)^{1+s}}=\frac{1}{\Gamma\left(\frac{1+s}{2}\right)} \int_{0}^{\infty} d t t^{\frac{s-1}{2}} e^{-t O^{2}}
$$

7 Here we assume that the spatial directions are infinitely large for simplicity. 
for a Hermitian operator $O$, our goal is to compute

$$
\begin{aligned}
\eta\left(H_{D W}\right)= & \lim _{s \rightarrow 0} \frac{1}{\Gamma\left(\frac{1+s}{2}\right)} \int_{0}^{\infty} d t t^{\frac{s-1}{2}} \lim _{M \rightarrow \infty} \operatorname{Tr}\left[\gamma_{5} \epsilon\left(x_{4}\right) e^{-t \frac{H_{D W}^{2}}{M^{2}}}\right] \\
& +\int_{0}^{1} d u \lim _{s \rightarrow 0} \frac{1}{\Gamma\left(\frac{1+s}{2}\right)} \int_{0}^{\infty} d t t^{\frac{s-1}{2}} \lim _{M \rightarrow \infty} \operatorname{Tr}\left[-s \frac{d H_{D W}(u) e^{-t \frac{H_{D W}(u)^{2}}{M^{2}}}}{M}\right] \\
& -\int_{0}^{1} d u \frac{d}{d u}\left\{\lim _{s \rightarrow 0} \frac{1}{\Gamma\left(\frac{1+s}{2}\right)} \int_{0}^{\infty} d t t^{\frac{s-1}{2}} \lim _{M \rightarrow \infty} \operatorname{Tr}\left[\gamma_{5} \epsilon\left(x_{4}\right) e^{-t \frac{H_{D W}(u)^{2}}{M^{2}}}\right]\right\},
\end{aligned}
$$

inserting our complete set $\left\{\phi_{\boldsymbol{p}, \uparrow \downarrow}^{3 \mathrm{D}}(\boldsymbol{x}) \otimes \varphi_{ \pm, e / o}^{\omega}\left(x_{4}\right)\right\}$, and $\left\{\phi_{\boldsymbol{p}, \uparrow \downarrow}^{3 \mathrm{D}}(\boldsymbol{x}) \otimes \varphi_{+, e}^{\text {edge }}\left(x_{4}\right)\right\}$ to the trace. To make $t$ dimensionless, we have rescaled $H_{D W}$ to $H_{D W} / M$. The third term can be easily evaluated once the first term is obtained.

1. The first term of Eq. 91,

Let us evaluate the first term in Eq. (91), using a general formula

$$
\begin{aligned}
\operatorname{Tr} f\left(\frac{H_{D W}^{2}}{M^{2}}\right)= & \int d^{4} x \sum_{g= \pm} \sum_{a=e, o} \int_{0}^{\infty} d \omega \sum_{\sigma=\uparrow \downarrow} \int d^{3} p \operatorname{tr}_{c}\left[\phi_{\boldsymbol{p}, \sigma}^{3 \mathrm{D}}(\boldsymbol{x}) \varphi_{g, a}^{\omega}\left(x_{4}\right)\right]^{\dagger} \\
& \times f\left(\frac{H_{D W}^{2}}{M^{2}}\right)\left[\phi_{\boldsymbol{p}, \sigma}^{3 \mathrm{D}}(\boldsymbol{x}) \varphi_{g, a}^{\omega}\left(x_{4}\right)\right] \\
& +\int d^{4} x \sum_{\sigma=\uparrow \downarrow} \int d^{3} p \operatorname{tr}_{c}\left\{\left[\phi_{\boldsymbol{p}, \sigma}^{3 \mathrm{D}}(\boldsymbol{x}) \varphi_{+, e}^{\text {edge }}\left(x_{4}\right)\right]^{\dagger} f\left(\frac{H_{D W}^{2}}{M^{2}}\right)\left[\phi_{\boldsymbol{p}, \sigma}^{3 \mathrm{D}}(\boldsymbol{x}) \varphi_{+, e}^{\mathrm{edge}}\left(x_{4}\right)\right]\right\} \\
= & \int d^{4} x \sum_{g= \pm} \sum_{a=e, o} \int_{0}^{\infty} d \omega \sum_{\sigma=\uparrow \downarrow} \int \frac{d^{3} p}{(2 \pi)^{3}} \operatorname{tr}_{c}\left\{\left[v_{\sigma} \varphi_{g, a}^{\omega}\left(x_{4}\right)\right]^{\dagger}\right. \\
& \left.\times f\left(1-\frac{\left(i p^{i} \gamma_{i}+D\right)^{2}+2 M \gamma_{4} \delta\left(x_{4}\right)}{M^{2}}\right)\left[v_{\sigma} \varphi_{g, a}^{\omega}\left(x_{4}\right)\right]\right\} \\
& +\int d^{4} x \sum_{\sigma=\uparrow \downarrow} \int \frac{d^{3} p}{(2 \pi)^{3}} \operatorname{tr}_{c}\left\{\left[v_{\sigma} \varphi_{+, e}^{\text {edge }}\left(x_{4}\right)\right]^{\dagger}\right. \\
& \left.\times f\left(1-\frac{\left(i p^{i} \gamma_{i}+D\right)^{2}+2 M \gamma_{4} \delta\left(x_{4}\right)}{M^{2}}\right)\left[v_{\sigma} \varphi_{+, e}^{\text {edge }}\left(x_{4}\right)\right]\right\}
\end{aligned}
$$

for any (finite) function $f$. We can see that in the expansion of $e^{-t \frac{H_{D W}^{2}}{M^{2}}}$ in $1 / M^{2}$, only the term proportional to $t^{2}$ and having four different gamma matrices can contribute to the 
trace. Namely, we only need to evaluate

$$
\begin{aligned}
\varphi_{g, a}^{\omega}( & \left.x_{4}\right)^{\dagger} \epsilon\left(x_{4}\right)\left[\gamma_{5} e^{-t\left(1-\frac{\left(i p^{i} \gamma_{i}+D\right)^{2}+2 M \gamma_{4} \delta\left(x_{4}\right)}{M^{2}}\right)}\right] \varphi_{g, a}^{\omega}\left(x_{4}\right)=\epsilon\left(x_{4}\right) \varphi_{g, a}^{\omega}\left(x_{4}\right)^{\dagger} e^{-\frac{t}{M^{2}}\left(\omega^{2}+p^{2}\right)-t} \\
\times & {\left[-\frac{t^{2}}{8 M^{4}} \gamma_{5}\left\{\left[\gamma_{i}, \gamma_{j}\right] \gamma_{4} \gamma_{k} F^{i j} F^{4 k}+\gamma_{4} \gamma_{k}\left[\gamma_{i}, \gamma_{j}\right] F^{4 k} F^{i j}\right\}\right] \varphi_{g, a}^{\omega}\left(x_{4}\right) } \\
= & -1_{2 \times 2} \epsilon\left(x_{4}\right) \varphi_{g, a}^{\omega}\left(x_{4}\right)^{\dagger} \varphi_{g, a}^{\omega}\left(x_{4}\right) e^{-\frac{t}{M^{2}}\left(\omega^{2}+\boldsymbol{p}^{2}\right)-t} \frac{t^{2}}{4 M^{4}} \epsilon_{i j k}\left(F^{i j} F^{4 k}+F^{4 k} F^{i j}\right),
\end{aligned}
$$

and similarly,

$$
\begin{aligned}
\varphi_{+, e}^{\text {edge }}\left(x_{4}\right)^{\dagger} \epsilon\left(x_{4}\right)\left[\gamma_{5} e^{-t\left(1-\frac{\left(i p^{i} \gamma_{i}+D\right)^{2}+2 M \gamma_{4} \delta\left(x_{4}\right)}{M^{2}}\right)}\right] \varphi_{+, e}^{\text {edge }}\left(x_{4}\right) \\
\quad=-1_{2 \times 2} \epsilon\left(x_{4}\right) \varphi_{+, e}^{\text {edge }}\left(x_{4}\right)^{\dagger} \varphi_{+, e}^{\text {edge }}\left(x_{4}\right) e^{-\frac{t}{M^{2}}\left(\boldsymbol{p}^{2}\right)} \frac{t^{2}}{4 M^{4}} \epsilon_{i j k}\left(F^{i j} F^{4 k}+F^{4 k} F^{i j}\right) .
\end{aligned}
$$

Then we have

$$
\operatorname{Tr}\left[\epsilon\left(x_{4}\right) \gamma_{5} e^{-t \frac{H_{D W}^{2}}{M^{2}}}\right]=-\int d^{4} x \epsilon\left(x_{4}\right) \frac{\sqrt{\pi t}}{8 \pi^{2}}\left(\frac{e^{-t}}{\sqrt{\pi t}}-h\left(t ; x_{4}, M\right)\right) \epsilon_{i j k} \operatorname{tr}_{c} F^{i j} F^{4 k} .
$$

Here

$$
h\left(t ; x_{4}, M\right)=\frac{1}{2} e^{2 M\left|x_{4}\right|} \operatorname{erfc}\left(\frac{M\left|x_{4}\right|}{\sqrt{t}}+\sqrt{t}\right)+\frac{1}{2} e^{-2 M\left|x_{4}\right|}\left[\operatorname{erfc}\left(-\frac{M\left|x_{4}\right|}{\sqrt{t}}+\sqrt{t}\right)-2\right]
$$

and we have used

$$
\begin{gathered}
\sum_{\sigma=\uparrow \downarrow} v_{\sigma}^{\dagger} v_{\sigma}=2 \\
\int \frac{d^{3} p}{(2 \pi)^{3}} e^{-t p^{2} / M^{2}}=\frac{M^{3} \sqrt{\pi}}{8 \pi^{2} t \sqrt{t}}
\end{gathered}
$$

and

$$
\begin{array}{r}
\sum_{a=e, o} \sum_{g= \pm} \int_{0}^{\infty} d \omega \varphi_{g, a}^{\omega}\left(x_{4}\right)^{\dagger} \varphi_{g, a}^{\omega}\left(x_{4}\right) e^{-\frac{t}{M^{2}}\left(\omega^{2}+M^{2}\right)}+\varphi_{+, e}^{\text {edge }}\left(x_{4}\right)^{\dagger} \varphi_{+, e}^{\text {edge }}\left(x_{4}\right) \\
=M\left[\frac{e^{-t}}{\sqrt{\pi t}}-\frac{1}{2} e^{2 M\left|x_{4}\right|} \operatorname{erfc}\left(\frac{M\left|x_{4}\right|}{\sqrt{t}}+\sqrt{t}\right)-\frac{1}{2} e^{-2 M\left|x_{4}\right|} \operatorname{erfc}\left(-\frac{M\left|x_{4}\right|}{\sqrt{t}}+\sqrt{t}\right)+1\right] .
\end{array}
$$

With the $t$ integrals

$$
\begin{gathered}
\lim _{s \rightarrow 0} \frac{1}{\Gamma\left(\frac{1+s}{2}\right)} \int_{0}^{\infty} d t t^{\frac{s-1}{2}} e^{-t}=1 \\
\lim _{s \rightarrow 0} \frac{1}{\Gamma\left(\frac{1+s}{2}\right)} \int_{0}^{\infty} d t t^{\frac{s-1}{2}} \sqrt{\pi t} e^{2 M\left|x_{4}\right|} \operatorname{erfc}\left(\frac{M\left|x_{4}\right|}{\sqrt{t}}+\sqrt{t}\right)=\frac{e^{-2 M\left|x_{4}\right|}}{2}
\end{gathered}
$$




$$
\lim _{s \rightarrow 0} \frac{\sqrt{\pi}}{\Gamma\left(\frac{1+s}{2}\right)} \int_{0}^{\infty} d t t^{\frac{s}{2}}\left[e^{-2 M\left|x_{4}\right|}\right]=\lim _{T \rightarrow \infty} T e^{-2 M\left|x_{4}\right|}
$$

and

$$
\lim _{s \rightarrow 0} \frac{\sqrt{\pi}}{\Gamma\left(\frac{1+s}{2}\right)} \int_{0}^{\infty} d t t^{\frac{s}{2}}\left[-e^{-2 M\left|x_{4}\right|} \operatorname{erfc}\left(\frac{-M\left|x_{4}\right|}{\sqrt{t}}+\sqrt{t}\right)\right]=-e^{-2 M\left|x_{4}\right|} \frac{1+4 M\left|x_{4}\right|}{2},
$$

we have

$$
g\left(x_{4}, M\right)=\lim _{s \rightarrow 0} \frac{1}{\Gamma\left(\frac{1+s}{2}\right)} \int_{0}^{\infty} d t t^{\frac{s-1}{2}} \sqrt{\pi t} h\left(t ; x_{4}, M\right)=\frac{e^{-2 M\left|x_{4}\right|}}{2}\left(1+2 M\left|x_{4}\right|-2 \lim _{T \rightarrow \infty} T\right),
$$

with which any finite function $f\left(x_{4}\right)$ gives

$$
\begin{aligned}
\lim _{M \rightarrow \infty} \int_{0}^{\infty} d x_{4} g\left(x_{4}, M\right) f\left(x_{4}\right) & <\lim _{M \rightarrow \infty} \int_{0}^{\infty} d x_{4} g\left(x_{4}, M\right)\left|f^{\max }\right| \\
& =\lim _{T \rightarrow \infty} \lim _{M \rightarrow \infty} \frac{\left|f^{\max }\right|(T-1)}{2 M} \rightarrow 0
\end{aligned}
$$

where $\left|f^{\max }\right|$ denotes the maximum of $f\left(x_{4}\right)$, and the same is true in the $x_{4}<0$ region. Therefore, we obtain the first term in Eq. (91) as

$$
\begin{aligned}
\lim _{s \rightarrow 0} \frac{1}{\Gamma\left(\frac{1+s}{2}\right)} \int_{0}^{\infty} d t t^{\frac{s-1}{2}} \lim _{M \rightarrow \infty} \operatorname{Tr}\left[\gamma_{5} \epsilon\left(x_{4}\right) e^{-t \frac{H_{D W}^{2}}{M^{2}}}\right] & =-\int d^{4} x \epsilon\left(x_{4}\right) \frac{1}{8 \pi^{2}} \epsilon_{i j k} \operatorname{tr}_{c} F^{i j} F^{4 k} \\
& =\frac{1}{32 \pi^{2}} \int d^{4} x \epsilon\left(x_{4}\right) \epsilon_{\mu \nu \rho \sigma} \operatorname{tr}_{c} F^{\mu \nu} F^{\rho \sigma}
\end{aligned}
$$

2. The third term of Eq. 91)

Noticing

$$
\begin{aligned}
& \frac{1}{32 \pi^{2}} \int_{x_{4}>0} d^{4} x \epsilon_{\mu \nu \rho \sigma} \operatorname{tr}_{c} F^{\mu \nu} F^{\rho \sigma}=\left.\frac{1}{2 \pi} C S\right|_{x_{4}=0}+\text { integer } \\
& \frac{1}{32 \pi^{2}} \int_{x_{4}<0} d^{4} x \epsilon_{\mu \nu \rho \sigma} \operatorname{tr}_{c} F^{\mu \nu} F^{\rho \sigma}=-\left.\frac{1}{2 \pi} C S\right|_{x_{4}=0}+\text { integer }
\end{aligned}
$$

we can compute the third term in Eq. 91 as

$$
-\int_{0}^{1} d u \frac{d}{d u}\left(\left.\frac{1}{\pi} C S^{u}\right|_{x_{4}=0}\right)=-\left.\frac{1}{\pi} C S\right|_{x_{4}=0},
$$

where $C S^{u}$ means the Chern-Simons term with the gauge field $u A_{\mu}$. 
3. The second term of Eq. 91.

For the second term in Eq. 91, only the linear term in $t$ in the exponential $e^{-t \frac{H_{D W}^{2}}{M^{2}}}$ with two different gamma matrices in spatial directions can contribute. Therefore, we need

$$
\begin{aligned}
\varphi_{g, a}^{\omega}\left(x_{4}\right)^{\dagger}\left[-s i \gamma_{5}\right. & \left.\gamma^{k} A_{k} e^{-t\left(1-\frac{\left(i p^{i} \gamma_{i}+D^{u}\right)^{2}+2 M \gamma_{4} \delta\left(x_{4}\right)}{M^{2}}\right)}\right] \varphi_{g, a}^{\omega}\left(x_{4}\right)=\varphi_{g, a}^{\omega}\left(x_{4}\right)^{\dagger} e^{-\frac{t}{M^{2}}\left(\omega^{2}+\boldsymbol{p}^{2}\right)-t} \\
\times & {\left[-\operatorname{si} \gamma_{5} \gamma^{k} A_{k} \frac{t}{M^{2}}\left\{\frac{i}{4}\left[\gamma_{i}, \gamma_{j}\right] F_{u}^{i j}\right\}\right] \varphi_{g, a}^{\omega}\left(x_{4}\right) } \\
& =-1_{2 \times 2}\left\{\varphi_{g, a}^{\omega}\left(x_{4}\right)^{\dagger}\left[\tau_{3}\right] \varphi_{g, a}^{\omega}\left(x_{4}\right)\right\} e^{-\frac{t}{M^{2}}\left(\omega^{2}+\boldsymbol{p}^{2}\right)-t} \frac{s t}{2 M^{2}} \epsilon_{i j k} A^{k} F_{u}^{i j},
\end{aligned}
$$

and similarly

$$
\begin{gathered}
\varphi_{+, e}^{\text {edge }}\left(x_{4}\right)^{\dagger}\left[-s i \gamma_{5} \gamma^{k} A_{k} e^{-t\left(1-\frac{\left(i p^{i} \gamma_{i}+D^{u}\right)^{2}+2 M \gamma_{4} \delta\left(x_{4}\right)}{M^{2}}\right)}\right] \varphi_{+, e}^{\text {edge }}\left(x_{4}\right) \\
=-1_{2 \times 2} \varphi_{+, e}^{\text {edge }}\left(x_{4}\right)^{\dagger} \varphi_{+, e}^{\text {edge }}\left(x_{4}\right) e^{-\frac{t}{M^{2}} p^{2}} \frac{s t}{2 M^{2}} \epsilon_{i j k} A^{k} F_{u}^{i j},
\end{gathered}
$$

where $D^{u}$ and $F_{u}^{i j}$ are the Dirac operator and field strength for the gauge field $u A$, respectively. Then we have

$$
\begin{aligned}
\operatorname{Tr}\left[-s \frac{d H_{D W}(u)}{d u} \frac{e^{-t \frac{H_{D W}(u)^{2}}{M^{2}}}}{M}\right]= & \int d^{4} x \frac{-s}{8 \pi^{2}} \frac{\partial}{\partial\left|x_{4}\right|}\left[\frac{\sqrt{\pi}}{4 \sqrt{t}} e^{-2 M\left|x_{4}\right|}\left\{\operatorname{erfc}\left(\frac{-M\left|x_{4}\right|}{\sqrt{t}}+\sqrt{t}\right)-2\right\}\right. \\
& \left.+\frac{\sqrt{\pi}}{4 \sqrt{t}} e^{2 M\left|x_{4}\right|} \operatorname{erfc}\left(\frac{M\left|x_{4}\right|}{\sqrt{t}}+\sqrt{t}\right)\right] \epsilon_{i j k} \operatorname{tr}_{c} A^{k} F_{u}^{i j},
\end{aligned}
$$

where we have used Eq. (97), Eq. (98), and

$$
\begin{array}{r}
\sum_{a=e, o} \sum_{g= \pm} \int_{0}^{\infty} d \omega \varphi_{g, a}^{\omega}\left(x_{4}\right)^{\dagger}\left[\tau_{3}\right] \varphi_{g^{\prime}, a}^{\omega}\left(x_{4}\right) e^{-\frac{t}{M^{2}}\left(\omega^{2}+M^{2}\right)}+\varphi_{+}^{\text {edge }}\left(x_{4}\right)^{\dagger} \varphi_{+}^{\text {edge }}\left(x_{4}\right) \\
=1_{2 \times 2} \frac{1}{4} \frac{\partial}{\partial\left|x_{4}\right|}\left[e^{-2 M\left|x_{4}\right|}\left\{\operatorname{erfc}\left(\frac{-M\left|x_{4}\right|}{\sqrt{t}}+\sqrt{t}\right)-2\right\}+e^{2 M\left|x_{4}\right|} \operatorname{erfc}\left(\frac{M\left|x_{4}\right|}{\sqrt{t}}+\sqrt{t}\right)\right] .
\end{array}
$$

Here let us compute

$$
\begin{aligned}
g_{1}\left(x_{4}, M\right) & =\frac{1}{\Gamma\left(\frac{1+s}{2}\right)} \int_{0}^{\infty} d t t^{\frac{s-1}{2}}\left[\frac{\sqrt{\pi}}{2 \sqrt{t}} e^{2 M\left|x_{4}\right|} \operatorname{erfc}\left(\frac{M\left|x_{4}\right|}{\sqrt{t}}+\sqrt{t}\right)\right] \\
& =-\frac{e^{2 M\left|x_{4}\right|}}{s \Gamma\left(\frac{1+s}{2}\right)} \int_{0}^{\infty} d t t^{\frac{s}{2}}\left[\left(\frac{M\left|x_{4}\right|}{t^{3 / 2}}-\frac{1}{t^{1 / 2}}\right) e^{-\left(M\left|x_{4}\right| / \sqrt{t}+\sqrt{t}\right)^{2}}\right] \\
& =-\frac{2\left(M\left|x_{4}\right|\right)^{(s+1) / 2}}{s \Gamma\left(\frac{1+s}{2}\right)}\left(K_{(s-1) / 2}\left(2 M\left|x_{4}\right|\right)-K_{(s+1) / 2}\left(2 M\left|x_{4}\right|\right)\right),
\end{aligned}
$$

where $K_{\nu}(z)$ are modified Bessel functions. $g_{1}\left(x_{4}, M\right)$ has the following properties.

$$
g_{1}(0, M)=\frac{1}{s}, \quad g_{1}\left(x_{4} \neq 0, M\right)=O(1)
$$


where we have used the expansion

$$
K_{\nu}(x)=x^{-\nu}\left(2^{\nu-1} \Gamma(\nu)+O\left(x^{2}\right)\right)+x^{\nu}\left(2^{-\nu-1} \Gamma(-\nu)+O\left(x^{2}\right)\right),
$$

for small $x$, and

$$
\int_{-\infty}^{\infty} d x_{4} \frac{\partial}{\partial\left|x_{4}\right|} g_{1}\left(x_{4}, M\right)=-\frac{2}{s} .
$$

Therefore, we can regard that

$$
\lim _{s \rightarrow 0} s \frac{\partial}{\partial\left|x_{4}\right|} g_{1}\left(x_{4}, M\right)=-2 \delta\left(x_{4}\right)
$$

Similarly

$$
\begin{aligned}
g_{2}\left(x_{4}, M\right) & =\frac{1}{\Gamma\left(\frac{1+s}{2}\right)} \int_{0}^{\infty} d t t^{\frac{s-1}{2}}\left[\frac{\sqrt{\pi}}{2 \sqrt{t}} e^{-2 M\left|x_{4}\right|}\left\{\operatorname{erfc}\left(\frac{-M\left|x_{4}\right|}{\sqrt{t}}+\sqrt{t}\right)-2\right\}\right] \\
& =-\frac{2 e^{-2 M\left|x_{4}\right|} \sqrt{\pi}}{s \Gamma\left(\frac{1+s}{2}\right)} \lim _{T \rightarrow \infty} T^{s / 2}+\frac{2\left(M\left|x_{4}\right|\right)^{\frac{s+1}{2}}}{s \Gamma\left(\frac{1+s}{2}\right)}\left(K_{\frac{s+1}{2}}\left(2 M\left|x_{4}\right|\right)+K_{\frac{s-1}{2}}\left(2 M\left|x_{4}\right|\right)\right)
\end{aligned}
$$

has the following properties,

$$
g_{2}(0, M)=-\frac{1}{s}, \quad g_{2}\left(x_{4} \neq 0, M\right)=O(1),
$$

and

$$
\int_{-\infty}^{\infty} d x_{4} \frac{\partial}{\partial\left|x_{4}\right|} g_{2}\left(x_{4}, M\right)=\frac{2}{s}+O(1)
$$

Therefore, we have

$$
\lim _{s \rightarrow 0} s \frac{\partial}{\partial\left|x_{4}\right|} g_{2}\left(x_{4}, M\right)=2 \delta\left(x_{4}\right) .
$$

Interestingly, the contribution from $g_{1}\left(x_{4}, M\right)$ and $g_{2}\left(x_{4}, M\right)$ cancels.

$$
\lim _{s \rightarrow 0} \frac{s}{2} \int_{-\infty}^{\infty} d x_{4} \frac{\partial}{\partial\left|x_{4}\right|}\left(g_{2}\left(x_{4}, M\right)+g_{1}\left(x_{4}, M\right)\right) f\left(x_{4}\right)=0
$$

so does the integrand of the second term in Eq. (91),

$$
\lim _{s \rightarrow 0} \frac{1}{\Gamma\left(\frac{1+s}{2}\right)} \int_{0}^{\infty} d t t^{\frac{s-1}{2}} \operatorname{Tr}\left[-s \frac{d H_{D W}(u)}{d u} \frac{e^{-t \frac{H_{D W}(u)^{2}}{M^{2}}}}{M}\right]=0,
$$

at finite $M$. 


\section{Final result of $\eta\left(H_{D W}\right)$}

Summing up all the contributions, we obtain

$$
\eta\left(H_{D W}\right)=\frac{1}{32 \pi^{2}} \int d^{4} x \epsilon\left(x_{4}\right) \epsilon_{\mu \nu \rho \sigma} \operatorname{tr}_{c} F^{\mu \nu} F^{\rho \sigma}-\eta\left(i D^{3 \mathrm{D}}\right),
$$

where we have added $2\left[\left.C S\right|_{x_{4}=0} / 2 \pi\right]$ according to the prescription in Eq. (60). The first term of Eq. 125) contains contribution only from the bulk modes; the edge-localized modes contribute to $g\left(x_{4}, M\right)$, which disappears in the large $M$ limit, whereas the second term of Eq. (125) entirely comes from the edge-localized modes, as explicitly computed in the previous subsection. In the above derivation, Eq. 124 is particularly important since it is equivalent to showing

$$
\frac{\partial \eta\left(H_{D W}(u)\right)}{\partial u}=0
$$

Moreover, we can also show

$$
\frac{\partial \eta\left(H_{D W}\right)}{\partial M}=-\lim _{s \rightarrow 0} \frac{s}{M} \frac{1}{32 \pi^{2}} \int d^{4} x \epsilon\left(x_{4}\right) \epsilon_{\mu \nu \rho \sigma} \operatorname{tr}_{c} F^{\mu \nu} F^{\rho \sigma}=0 .
$$

Namely, our definition of the index is stable against any variational changes in $M$ and gauge field $A_{\mu}$. It only allows discrete jumps by an even integer in the boundary contribution $\eta\left(i D^{3 \mathrm{D}}\right)$.

\section{APS index and physical interpretation}

We have shown that the index is equivalent to that of APS, i.e.

$$
\mathcal{I}_{x_{4}>0}=\frac{1}{2} \eta\left(H_{D W}\right)-\frac{1}{2} \eta\left(H_{P V}\right)=\frac{1}{32 \pi^{2}} \int_{x_{4}>0} d^{4} x \epsilon_{\mu \nu \rho \sigma} \operatorname{tr}_{c} F^{\mu \nu} F^{\rho \sigma}-\frac{\eta\left(i D^{3 \mathrm{D}}\right)}{2} .
$$

If we flip the sign of the Pauli-Villars mass, we obtain the same index in the $x_{4}<0$ region:

$$
-\mathcal{I}_{x_{4}<0}=\frac{1}{2} \eta\left(H_{D W}\right)-\frac{1}{2} \eta\left(\left.H_{P V}\right|_{M \rightarrow-M}\right)=-\frac{1}{32 \pi^{2}} \int_{x_{4}<0} d^{4} x \epsilon_{\mu \nu \rho \sigma} \operatorname{tr}_{c} F^{\mu \nu} F^{\rho \sigma}-\frac{\eta\left(i D^{3 \mathrm{D}}\right)}{2} .
$$

In fact, the eta invariant of the domain-wall fermion Dirac operator can be written as the difference between the APS indices in the two regions:

$$
\eta\left(H_{D W}\right)=\mathcal{I}_{x_{4}>0}-\mathcal{I}_{x_{4}<0} .
$$


Then the sign of the Pauli-Villars mass determines which is topological and which is normal insulator. In our computations, we do not need the massless Dirac operator or global boundary conditions on the fermion fields. Moreover, we have seen that the eta invariant $\eta\left(i D^{3 \mathrm{D}}\right)$ comes entirely from the edge-localized modes, while these edge modes do not contribute to the first term of Eq. 128 at all.

As a final remark of this section, let us consider the second domain-wall or anti-domain wall, which is needed to compactify our set-up with flat metric. To define the index, we formally need to consider the domain-wall Dirac fermion operator in a finite region of $-L<$ $x_{4}<L$, and, for example, identify the fermion field at $x_{4}=L$ and $-L$ (periodic boundary condition):

$$
H_{D W}=\gamma_{5}\left\{D+M \epsilon\left(x_{4}\right) \epsilon\left(L-x_{4}\right)\right\}
$$

where the spatial directions are also required to be compactified. Even in this case, our computation above is valid, at least, in the large volume limit in the near region of $x_{4}=0$ and it should be naturally and smoothly continuated to the another domain-wall at $x_{4}=L$. Finally we obtain

$$
\begin{aligned}
\mathcal{I}=\frac{1}{2} \eta\left(H_{D W}\right)-\frac{1}{2} \eta\left(H_{P V}\right)= & \frac{1}{32 \pi^{2}} \int_{0<x_{4}<L} d^{4} x \epsilon_{\mu \nu \rho \sigma} \operatorname{tr}_{c} F^{\mu \nu} F^{\rho \sigma} \\
& -\left.\frac{\eta\left(i D^{3 \mathrm{D}}\right)}{2}\right|_{x_{4}=0}+\left.\frac{\eta\left(i D^{3 \mathrm{D}}\right)}{2}\right|_{x_{4}=L} .
\end{aligned}
$$

\section{ASYMMETRIC DOMAIN-WALL}

In the previous section, we have considered the domain-wall fermion Dirac operator from which the APS index has been reproduced. Although the domain-wall fermion is a good model to describe the topological insulator with boundary, the size of the fermion gap $|M|$ is the same both in the normal and topological phases, which is not generally true in the actual materials. In this section, we consider a more general case where the two regions $x_{4}<0$ and $x_{4}>0$ have different mass gaps.

\section{A. Effect of asymmetric mass}

Let us consider a modified model with an additional mass $M_{2}$ without the kink structure,

$$
H_{D W}=\gamma_{5}\left(D+M_{1} \epsilon\left(x_{4}\right)-M_{2}\right)
$$


where both $M_{1}$ and $M_{2}$ are positive. This introduces the asymmetric mass to the fermion in normal and topological phases, and a step-function-like term in $H_{D W}^{2}$ (when $\left.\partial_{x_{4}} B=0\right)$,

$$
H_{D W}^{2}=-\partial_{4}^{2}+B^{2}+M_{1}^{2}+M_{2}^{2}-2 M_{1} \gamma_{4} \delta\left(x_{4}\right)-2 M_{1} M_{2} \epsilon\left(x_{4}\right) .
$$

Because of the step function, there are three types of eigensolutions of $H_{W}^{2}$ using the same decomposition $\varphi_{ \pm}\left(x_{4}\right) \otimes \phi_{\lambda}^{3 \mathrm{D}}(\vec{x})$, where $i D^{3 \mathrm{D}} \phi_{\lambda}^{3 \mathrm{D}}(\vec{x})=\lambda \phi_{\lambda}^{3 \mathrm{D}}(\vec{x})$ and $\tau_{3} \varphi_{ \pm}\left(x_{4}\right)= \pm \varphi_{ \pm}\left(x_{4}\right)$ as in the previous section; 1) localized bound state (edge state),

$$
\varphi_{+}^{\text {edge }}\left(x_{4}\right)=\left\{\begin{array}{cc}
u_{+} \sqrt{\frac{M_{1}^{2}-M_{2}^{2}}{M_{1}}} e^{-\left(M_{1}-M_{2}\right) x_{4}} & \left(x_{4} \geq 0\right) \\
u_{+} \sqrt{\frac{M_{1}^{2}-M_{2}^{2}}{M_{1}}} e^{\left(M_{1}+M_{2}\right) x_{4}} & \left(x_{4}<0\right)
\end{array},\right.
$$

where the eigenvalue of $H_{W}^{2}$ is $\left.\Lambda^{2}=\lambda^{2}, 2\right)$ plane waves extended only in the $x_{4}>0$ region,

$$
\varphi_{ \pm}^{\omega}\left(x_{4}\right)=\left\{\begin{array}{cc}
\frac{u_{ \pm}}{\sqrt{2 \pi\left(\omega^{2}+\mu_{ \pm}^{2}\right)}}\left\{\left(i \omega+\mu_{ \pm}\right) e^{i \omega x_{4}}+\left(i \omega-\mu_{ \pm}\right) e^{-i \omega x_{4}}\right\} & \left(x_{4} \geq 0\right) \\
u_{ \pm} \frac{2 i \omega}{\sqrt{2 \pi\left(\omega^{2}+\mu_{ \pm}^{2}\right)}} e^{\Omega x_{4}} & \left(x_{4}<0\right)
\end{array}\right.
$$

where $\omega=\sqrt{\Lambda^{2}-\lambda^{2}-\left(M_{1}-M_{2}\right)^{2}}, \Omega=\sqrt{-\Lambda^{2}+\lambda^{2}+\left(M_{1}+M_{2}\right)^{2}}$, and $\mu_{ \pm}=\Omega \mp 2 M_{1}$, and 3) plane waves extended in the whole region.

$$
\varphi_{ \pm}^{\omega}\left(x_{4}\right)=\left\{\begin{array}{ll}
u_{ \pm}\left(A e^{i \omega_{1} x_{4}}+B e^{-i \omega_{1} x_{4}}\right) & \left(x_{4} \geq 0\right) \\
u_{ \pm}\left(C e^{i \omega_{2} x_{4}}+D e^{-i \omega_{2} x_{4}}\right) & \left(x_{4}<0\right)
\end{array},\right.
$$

where $\omega_{1}=\sqrt{\Lambda^{2}-\lambda^{2}-\left(M_{1}-M_{2}\right)^{2}}, \omega_{2}=\sqrt{\Lambda^{2}-\lambda^{2}-\left(M_{1}+M_{2}\right)^{2}}$, and the coefficients satisfy $A+B=C+D$, and $-i \omega_{1}(A-B)+i \omega_{2}(C-D) \mp 2 M_{1}(A+B)=0$. The orthonormality of the above eigenfunctions can be confirmed using the relation $[39]^{8}$

$$
\int_{0}^{\infty} d x_{4} e^{i \omega x_{4}}=\pi \delta(\omega)+i \mathcal{P} \frac{1}{\omega}
$$

where $\mathcal{P}$ denotes the principal value.

It is important to note that the above solutions all satisfy the nontrivial boundary condition

$$
-\lim _{\epsilon \rightarrow 0}\left(\partial_{x_{4}} \varphi_{ \pm}^{\omega / \text { edge }}(+\epsilon)-\partial_{x_{4}} \varphi_{ \pm}^{\omega / \text { edge }}(-\epsilon)\right) \mp 2 M_{1} \varphi_{ \pm}^{\omega / \text { edge }}(0)=0
$$

which respects the $S O(3)$ rotational symmetry on the surface. It is also important to note that the edge mode exists only when $M_{1}>M_{2}$, otherwise the above solution is not normalizable.

8 We thank H. Nakazato and M. Ochiai for useful information about the system in a step potential. 
An appropriate Pauli-Villars operator in the case of $M_{1}>M_{2}$ is given by

$$
H_{P V}=\gamma_{5}\left(D-M_{1}+M_{2} \epsilon\left(x_{4}\right)\right)
$$

whose total mass $-M_{1}+M_{2} \epsilon\left(x_{4}\right)$ does not change its sign at $x_{4}$ and hence does not develop any edge-localized modes.

Since the additional mass $M_{2}$ does not break the $\gamma_{5}$ Hermiticity of the domain-wall and Pauli-Villars Dirac operators, we can define the index

$$
\mathcal{I}=\frac{1}{2} \eta\left(H_{D W}\right)-\frac{1}{2} \eta\left(H_{P V}\right)
$$

Furthermore, we can show

$$
\frac{d \mathcal{I}}{d M_{2}}=\lim _{s \rightarrow 0} s \times(\text { finite terms })=0
$$

for $M_{1}>M_{2}$, from which there is no doubt that $\mathcal{I}$ is equivalent to the APS index. It is still instructive to directly compute the index in an extreme case, where the mass gap in the $x_{4}<0$ region is infinitely large, and all the wave functions are constrained to the $x_{4} \geq 0$ region. This is equivalent to considering the original system of manifold with boundary, as a closed system (similar studies were done in Refs. [40, 41]).

\section{B. Shamir-type domain-wall}

In the following, let us take an extreme limit where $M_{1}+M_{2}=\infty$, while $M_{1}-M_{2}=M$ is fixed. In this case, we can safely neglect the type 3) plane wave solutions in Eq. (137) and the other two types of eigenfunctions become

$$
\varphi_{+}^{\text {edge }}\left(x_{4}\right)=\left\{\begin{array}{cc}
u_{+} \sqrt{2 M} e^{-M x_{4}} & \left(x_{4} \geq 0\right) \\
0 & \left(x_{4}<0\right)
\end{array},\right.
$$

where $\Lambda^{2}=\lambda^{2}$ is unchanged, and

$$
\begin{gathered}
\varphi_{+}^{\omega}\left(x_{4}\right)=\left\{\begin{array}{cc}
\frac{u_{+}}{\sqrt{2 \pi\left(\omega^{2}+M^{2}\right)}}\left\{(i \omega-M) e^{i \omega x_{4}}+(i \omega+M) e^{-i \omega x_{4}}\right\} & \left(x_{4} \geq 0\right) \\
0 & \left(x_{4}<0\right)
\end{array},\right. \\
\varphi_{-}^{\omega}\left(x_{4}\right)=\left\{\begin{array}{cc}
\frac{u_{-}}{\sqrt{2 \pi}}\left(e^{i \omega x_{4}}-e^{-i \omega x_{4}}\right) & \left(x_{4} \geq 0\right) \\
0 & \left(x_{4}<0\right)
\end{array}\right.
\end{gathered}
$$


where $\omega=\sqrt{\Lambda^{2}-\lambda^{2}-M^{2}}$. The above wave functions are equivalent to the complete set of the massive Dirac operator,

$$
H_{+}=\gamma_{5}(D+M)
$$

extending only in the $x_{4} \geq 0$ region, whose boundary condition is locally given by

$$
\left.\varphi_{-}\right|_{x_{4}=0}=0,\left.\quad\left(\partial_{x_{4}}+M\right) \varphi_{+}\right|_{x_{4}=0}=0 .
$$

In fact, this system corresponds to the so-called Shamir-type domain-wall fermion [32, 33].

In the same way, the complete set of the Pauli-Villars operator converges to that of

$$
H_{-}=\gamma_{5}(D-M)
$$

which are given by

$$
\begin{aligned}
\varphi_{P V+}^{\omega}\left(x_{4}\right) & =\frac{u_{+}}{\sqrt{2 \pi\left(\omega^{2}+M^{2}\right)}}\left\{(i \omega+M) e^{i \omega x_{4}}+(i \omega-M) e^{-i \omega x_{4}}\right\} \quad\left(x_{4} \geq 0\right) \\
\varphi_{P V-}^{\omega}\left(x_{4}\right) & =\frac{u_{-}}{\sqrt{2 \pi}}\left(e^{i \omega x_{4}}-e^{-i \omega x_{4}}\right) \quad\left(x_{4} \geq 0\right)
\end{aligned}
$$

satisfying another local boundary condition

$$
\left.\varphi_{P V-}\right|_{x_{4}=0}=0,\left.\quad\left(\partial_{x_{4}}-M\right) \varphi_{P V+}\right|_{x_{4}=0}=0 .
$$

Now we can explicitly compute with these complete sets the index

$$
\mathcal{I}=\frac{1}{2} \eta\left(H_{+}\right)-\frac{1}{2} \eta\left(H_{-}\right)
$$

In fact, except that the variation of the eta invariants does not vanish separately,

$$
\begin{aligned}
\int_{0}^{1} d u \frac{d}{d u} \eta\left(H_{ \pm}\right) & =\int_{0}^{1} d u \lim _{s \rightarrow 0} \frac{1}{\Gamma\left(\frac{1+s}{2}\right)} \int_{0}^{\infty} d t t^{\frac{s-1}{2}} \operatorname{Tr}\left[\mp s \frac{d H_{ \pm}(u)}{d u} \frac{e^{-t \frac{H_{ \pm}(u)^{2}}{M^{2}}}}{M}\right] \\
& =\mp \lim _{s \rightarrow 0} \int_{0}^{1} d u \int_{x_{4}>0} d^{4} x \frac{s}{8 \pi^{2}} \frac{\partial}{\partial x_{4}} g_{ \pm}\left(x_{4}, M\right) \epsilon_{i j k} \operatorname{tr}_{c} A^{k} F_{u}^{i j} \\
& =\mp \lim _{s \rightarrow 0} \int_{0}^{1} d u \lim _{\epsilon \rightarrow 0} \int_{x_{4}>0} d^{4} x \frac{s}{8 \pi^{2}}\left( \pm \frac{\delta\left(x_{4}-\epsilon\right)}{s}\right) \epsilon_{i j k} \operatorname{tr}_{c} A^{k} F_{u}^{i j} \\
& =-\left.\frac{C S}{2 \pi}\right|_{x_{4}=0},
\end{aligned}
$$


where $g_{+}\left(x_{4}, M\right)=g_{1}\left(x_{4}, M\right)$, and $g_{-}\left(x_{4}, M\right)=g_{2}\left(x_{4}, M\right)$, already appeared in Eqs. (114) and (119), respectively, the computation is very similar to the one obtained in the previous section. The results are summarized as

$$
\begin{aligned}
& \eta\left(H_{+}\right)=\frac{1}{32 \pi^{2}} \int_{x_{4}>0} d^{4} x \epsilon_{\mu \nu \rho \sigma} \operatorname{tr}_{c} F^{\mu \nu} F^{\rho \sigma}-\left.\frac{1}{\pi} C S\right|_{x_{4}=0}+2\left[\left.\frac{1}{2 \pi} C S\right|_{x_{4}=0}\right], \\
& \eta\left(H_{-}\right)=-\frac{1}{32 \pi^{2}} \int_{x_{4}>0} d^{4} x \epsilon_{\mu \nu \rho \sigma} \operatorname{tr}_{c} F^{\mu \nu} F^{\rho \sigma},
\end{aligned}
$$

where we have again put the Gauss symbol term $2\left[\left.C S\right|_{x_{4}=0} / 2 \pi\right]$ to maintain the gauge invariance. Thus, $\mathcal{I}$ turns out to be the same index as the original APS in Eq. (152).

\section{SUMMARY AND DISCUSSION}

In this work, we have tried to describe the APS index theorem in a "physicist-friendly" way in a simple set-up with a flat metric, for the Dirac fermion operator with $U(1)$ or $S U(N)$ gauge field background. Our method corresponds to a generalization of the Fujikawa method on closed manifolds to that on manifolds with boundaries.

First, we have revisited the original set-up by APS and reproduced the index theorem in an adiabatic expansion. Contrary to the intuition that the eta invariant is a contribution of the edge-localized modes, we have found that the APS boundary condition allows no such edge modes to exist. Instead, a non-trivial pole structure of the coefficients of the bulk extended modes produces it. We have also discussed that the APS boundary is unnatural and unlikely to be realized in actual materials with boundary.

Then we have discussed what is required in more physical set-ups. In physics, what we call boundary is actually a domain-wall on which some physical parameter becomes discontinuous. Every topological insulator is non-trivial only when it is surrounded by normal insulators. It is more natural to consider a massive fermion since it is not the helicity but rotational symmetry that should be preserved on a surface. Any boundary condition should not be imposed by hand but should be given by the local dynamics of the system. We have concluded that the domain-wall Dirac fermion operator is a good candidate to reformulate the index theorem in physics.

Next, we have defined a new index by the eta invariant of the four-dimensional domainwall fermion Dirac operator with its Pauli-Villars regulator. The kink structure in the mass term automatically forces the fermion fields to satisfy a boundary condition, which is locally 
given and respects the $S O(3)$ rotational symmetry on the surface. As a consequence, the edge-localized modes appear in the complete set of the free Dirac operator. We have applied the Fujikawa method to this complete set satisfying the non-trivial boundary condition. Since the boundary condition is no longer dependent of gauge fields, we do not need the adiabatic approximation. We have obtained an index, which is stable against the changes of mass and gauge field. This new index coincides with the APS index. Moreover, in our set-up, the physical origin of the eta invariant is clearer. It comes entirely from the edge-localized modes.

Finally, we have considered the case with asymmetric masses and computed the index in the limit where one of the masses goes to infinity. This case is closer to the original set-up by APS, where we do not need to consider the $x_{4}<0$ region. In lattice gauge theory, this extremal case is known as the Shamir-type domain-wall fermion. We have confirmed by the direct computation that the index remains the same as the original APS index.

In this work, we have employed the Pauli-Villars regularization. It is interesting to give a non-perturbative definition of the APS index based on the lattice regularization as was done for the AS index [42]. As the Wilson fermion Dirac operator has the $\gamma_{5}$ Hermiticity and its determinant is real, we would be able to define an index by $\eta\left(\gamma_{5}\left(D_{W}+M \epsilon\left(x_{4}\right)\right)\right) / 2$ (assuming that the Wilson term has the opposite sign to the mass $M$ ), which coincides with the APS index, at least, in the continuum limit. In the lattice regularization, one would be able to increase the effective number of flavors $N_{f}$, by tuning the mass and Wilson term, so that some of the doubler modes become physical [43]. Even in that case, the APS index would be unchanged except for the overall multiplication of $N_{f}$.

The APS index theorem describes a part of the anomaly descent equations [8 12], in which the parity anomaly or the CS term in $2 n+1$ dimensions appears as the surface term of the axial $U(1)$ anomaly in $2 n+2$ dimensions. Our work describing the same index in terms of the domain-wall Dirac operator corresponds to its fermionic expression. It is interesting to extend our work to the $2 n$-dimensional Weyl fermion system, which appears as the edgelocalized state of the $2 n+1$-dimensional gapped bulk fermions. As already investigated in the literature [44 46], the gauge anomaly should be canceled by the surface contribution from the bulk $\eta$-invariant.

A further interesting question is whether we can incorporate the full set of anomaly descent equations in the $2 n+2 \rightarrow 2 n+1 \rightarrow 2 n$ dimensions, in one Dirac fermion operator. 


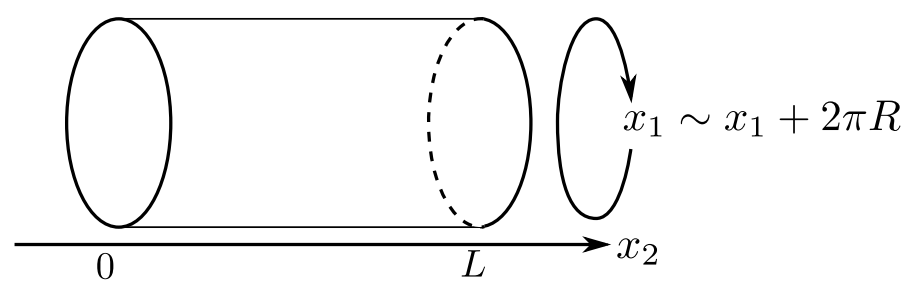

FIG. 1: two-dimensional cylinder.

In the conventional approach with the manifold with boundary, this is impossible since the boundary of the boundary must be trivial as a consequence of the homology. Combining two domain-walls having different quantum numbers, however, we have already proposed such an interesting "doubly gapped" fermion system [27, where the edge-of-edge state [47] appears only at the junction of the domain-walls. Our one-loop level computation shows that the structure of the full set of anomaly descent equations is embedded in the fermion determinant. The current work would provide a mathematical basis for investigating such non-trivial domain-wall systems.

\section{Acknowledgments}

We thank K. Hashimoto for organizing the study group on topological insulators and useful discussions. We also thank S. Aoki, M. Lüscher, T. Misumi, H. Suzuki and A. Tanaka for discussions. This work is supported in part by the Japanese Grant-in-Aid for Scientific Research(Grants No. JP26247043 and No. JP15K05054).

\section{Appendix A: Example in two dimensions}

In this appendix, we consider an example of $U(1)$ gauge theory in two dimensions with boundary. Under a constant background magnetic field, we non-perturbatively confirm the APS index theorem, discussed in Sec. II.

Let us consider a two-dimensional cylinder parameterized by $\left(x_{1}, x_{2}\right)$ as depicted in Fig. 1. Here $x_{1},\left(x_{1} \sim x_{1}+2 \pi R\right)$ parametrizes the circle of radius $R$, while $x_{2},\left(0 \leq x_{2} \leq L\right)$ parametrizes a segment of length $L$. This cylinder has two disconnected circular boundaries at $x_{2}=0$ and $x_{2}=L$.

We introduce a constant magnetic field $F_{12}=B$ on this cylinder. We choose the Landau 
gauge and the vector potential is written as

$$
A_{1}=-B x_{2}+\frac{a_{0}}{R}, \quad A_{2}=0
$$

where $a_{0}$ is a constant. This constant $a_{0}$ is the holonomy around the circle at $x_{2}=0$ boundary, which corresponds to the Chern-Simons term in one dimension:

$$
\frac{1}{2 \pi} \int d x_{1} A_{1}\left(x_{1}, x_{2}=0\right)=a_{0} \quad\left(=\frac{C S}{2 \pi}\right) .
$$

Similarly we define the holonomy $a_{L}$ at $x_{2}=L$ by

$$
\frac{1}{2 \pi} \int d x_{1} A_{1}\left(x_{1}, x_{2}=L\right)=: a_{L}
$$

A useful relation obtained from Eq. A1) using the Stoke's theorem is

$$
a_{L}=-\frac{1}{2 \pi} \int d^{2} x F_{12}+a_{0}
$$

We consider the Dirac operator on this cylinder given by

$$
D^{2 \mathrm{D}}=\gamma_{1} D_{1}+\gamma_{2} D_{2}
$$

where $\gamma_{i},(i=1,2)$ are $2 \times 2$ gamma matrices of two dimensions which satisfy $\left\{\gamma_{i}, \gamma_{j}\right\}=2 \delta_{i j}$, and $D_{i}=\partial_{i}+i A_{i},(i=1,2)$ are covariant derivatives. We also introduce the chirality matrix $\gamma_{3}$ by $\gamma_{3}=i \gamma_{1} \gamma_{2}$.

\section{APS index theorem in the two-dimensional example}

We count the index

$$
\operatorname{ind}\left(D^{2 \mathrm{D}}\right):=n_{+}-n_{-}, \quad n_{ \pm}:=\text {(number of zero-modes with } \gamma_{3}= \pm 1 \text { ), }
$$

by explicitly constructing the zero-mode wave functions under the APS boundary condition and confirm that the APS index theorem holds.

Since we have translation symmetry in the $x_{1}$ direction we can write the zero-mode wave functions as

$$
\psi_{n \pm}\left(x_{1}, x_{2}\right)=e^{i \frac{n}{R} x_{1}} \phi_{n \pm}\left(x_{2}\right), \quad n \in \mathbb{Z}
$$


where \pm stands for the chirality, i.e. $\gamma_{3} \psi_{n \pm}= \pm \psi_{n \pm}$ and $\gamma_{3} \phi_{n \pm}= \pm \phi_{n \pm}$. Then the zero-mode equation $D^{2 \mathrm{D}} \psi_{n \pm}=0$ implies

$$
\phi_{n \pm}\left(x_{2}\right)=\exp \left[ \pm \frac{1}{2 B}\left(B x_{2}-\frac{n+a_{0}}{R}\right)^{2}\right] \chi_{n \pm}
$$

where $\chi_{n \pm}$ is a constant spinor which satisfies $\gamma_{3} \chi_{n \pm}= \pm \chi_{n \pm}$. Thus once $n$ and the chirality \pm is given, the zero-mode wave function, if ever exists, is fixed up to overall constant.

Let us next consider the boundary condition at $x_{2}=0$. This boundary condition is $\psi\left(x_{2}=0\right)=0$ if eigenvalue of $\left.i \gamma_{3} D_{1}\right|_{x_{2}=0}$ is positive. Notice that if a zero-mode eigenfunction $\psi_{n \pm}(x)$ satisfy $\psi_{n \pm}\left(x_{2}=0\right)=0$ then $\psi_{n \pm}(x)=0$ for all $x_{2}$ as seen from Eq. A8). Therefore the zero-modes which survive after imposing the APS boundary condition at $x_{2}=0$ are

$$
\psi_{n+},\left(n+a_{0}>0\right), \quad \psi_{n-},\left(n+a_{0}<0\right) .
$$

Let us turn to the APS boundary condition at $x_{2}=L$. Since the orientation is opposite, the APS boundary condition is $\psi\left(x_{2}=L\right)=0$ if the eigenvalue of $-\left.i \gamma_{3} D_{1}\right|_{x_{2}=L}$ is positive. The surviving zero-modes are

$$
\psi_{n+},\left(n+a_{L}<0\right), \quad \psi_{n-},\left(n+a_{L}>0\right) .
$$

Finally let us combine both conditions eqs. A9, A10). The surviving zero-modes are

$$
\psi_{n+},\left(-a_{0}<n<-a_{L}\right), \quad \psi_{n-},\left(-a_{L}<n<-a_{0}\right) .
$$

As a result the number of zero-modes $n_{ \pm}$is given by

$$
\begin{aligned}
& n_{+}=\text {(number of integers } n, \quad-a_{0}<n<-a_{L} \text { ), } \\
& n_{-}=\text {(number of integers } n, \quad-a_{L}<n<-a_{0} \text { ). }
\end{aligned}
$$

When $B>0$ the inequality $-a_{0}<-a_{L}$ holds from Eq. (A4) and the numbers of zeromodes $n_{ \pm}$given in Eq. A12 read

$$
n_{+}=\left[a_{0}\right]-\left[a_{L}\right], \quad n_{-}=0,
$$

where [.] is the Gauss symbol. The index is rewritten by making use of Eq. (A4) as

$$
\operatorname{ind}\left(D^{2 \mathrm{D}}\right)=n_{+}-n_{-}=\left[a_{0}\right]-\left[a_{L}\right]=-a_{0}+a_{L}+\frac{1}{2 \pi} \int d^{2} x F_{12}+\left[a_{0}\right]-\left[a_{L}\right] .
$$


As we will see in Eqs. A26 and A34, the one-dimensional eta invariants are written in terms of $a_{0}, a_{L}$ as

$$
\eta\left(\left.i D_{1}\right|_{x_{2}=0}, 0\right)=-1+2\left(a_{0}-\left[a_{0}\right]\right), \quad \eta\left(\left.i D_{1}\right|_{x_{2}=L}, 0\right)=-1+2\left(a_{L}-\left[a_{L}\right]\right) .
$$

Substituting Eq. A15 into Eq. A14, we obtain

$$
\operatorname{ind}\left(D^{2 \mathrm{D}}\right)=\frac{1}{2 \pi} \int d^{2} x F_{12}-\frac{1}{2}\left(\eta\left(\left.i D_{1}\right|_{x_{2}=0}, 0\right)-\eta\left(\left.i D_{1}\right|_{x_{2}=L}, 0\right)\right) .
$$

This is nothing but the APS index theorem. Note that the result is unchanged even for $B<0$.

Let us mention an interesting observation about the zero-mode wave function. The condition A11 derived from APS boundary condition is equivalent to requiring that the wave function $\mathrm{A} 8 \mathrm{C}$ is Gaussian and the peak of this Gaussian wave function sits between two boundaries. This observation may be a hint to find a physical interpretation of the APS boundary condition.

\section{Eta invariant in 1 dimension}

We consider one-dimensional circle parametrized by $x_{1} \sim x_{1}+2 \pi R$ and the $U(1)$ gauge field on it. The one-dimensional Dirac operator is a simple covariant derivative, which is written as

$$
D^{1 \mathrm{D}}=\partial_{1}+i A_{1}
$$

We choose the gauge in which $A_{1}$ is a constant, whose integral gives a non-trivial ChernSimons term in one dimension:

$$
A_{1}=\frac{a}{R} \Rightarrow \oint A_{1} d y=2 \pi R A_{1}=2 \pi a(=C S) .
$$

The eigenvalues of $-i D^{1 \mathrm{D}}$ are

$$
\lambda_{n}=\frac{n+a}{R}, \quad n \in \mathbb{Z}
$$

where the $n$-th eigen-function is given by $e^{i n x_{1}}$. The eta invariant (with finite $s$ ) is defined as

$$
\eta\left(-i D^{1 \mathrm{D}}, s\right)=\sum_{n \in \mathbb{Z}} \operatorname{sign}\left(\lambda_{n}\right) \frac{1}{\left|\lambda_{n}\right|^{s}},
$$

and we take the $s \rightarrow 0$ limit. 


\section{a. Evaluation by Hurwitz zeta function}

If $a$ is not an integer, then by a large gauge transformation $a$ can be chosen such that

$$
0<a<1
$$

In this gauge choice the eta invariant $\mathrm{A} 20$ becomes

$$
\eta\left(-i D^{1 \mathrm{D}}, s\right)=\sum_{n=0}^{\infty} \frac{1}{(n+a)^{s}}-\sum_{n=-1}^{-\infty} \frac{1}{(-(n+a))^{s}}=\zeta(s, a)-\zeta(s, 1-a)
$$

where $\zeta(s, a)$ is the Hurwitz zeta function given for $\operatorname{Re} s>1$ by

$$
\zeta(s, a)=\sum_{n=0}^{\infty} \frac{1}{(n+a)^{s}}
$$

$\zeta(s, a)$ for $\operatorname{Re} s \leq 1$ is defined by the analytic continuation from $\operatorname{Re} s>1$.

It is known that for $a>0$ (see for example, Ch.12 of [48])

$$
\zeta(0, a)=\frac{1}{2}-a
$$

Since we choose the gauge in which $a>0,1-a>0$ we can apply this equation to A22 and obtain

$$
\eta\left(-i D^{1 \mathrm{D}}, 0\right)=1-2 a
$$

For a generic gauge, the eta invariant is written as

$$
\eta\left(-i D^{1 \mathrm{D}}, 0\right)=1-2(a-[a]), \quad a=\frac{1}{2 \pi} \oint A
$$

where [.] is the Gauss symbol. Notice that $\eta\left(-i D^{1 \mathrm{D}}, 0\right)$ is gauge invariant as we expected, although $a$ is not gauge invariant. In the prescription by APS, the zero eigenvalue is considered to be "positive". Therefore, the $a \rightarrow 0$ limit should be taken from the positive side, leading to $\left.\eta\left(-i D^{1 \mathrm{D}}, 0\right)\right|_{a \rightarrow 0} \rightarrow 1$. 


\section{b. Manifestly gauge invariant calculation}

Here we show more explicit and manifestly gauge invariant calculation of the eta invariant. We may rewrite $\eta\left(-i D^{1 \mathrm{D}}, s\right)$ in the following way.

$$
\begin{aligned}
\eta\left(-i D^{1 \mathrm{D}}, s\right) & =\sum_{n \in \mathbb{Z}} \frac{\lambda_{n}}{\left|\lambda_{n}\right|^{1+s}} \\
& =\frac{1}{\Gamma\left(\frac{s+1}{2}\right)} \int_{0}^{\infty} d t t^{\frac{s-1}{2}} \sum_{n \in \mathbb{Z}} \lambda_{n} e^{-t \lambda_{n}^{2}} \\
& =\frac{1}{\Gamma\left(\frac{s+1}{2}\right)} \int_{0}^{\infty} d t t^{\frac{s-1}{2}} \sum_{n \in \mathbb{Z}} \frac{(n+a)}{R} e^{-t\left(\frac{n+a}{R}\right)^{2}} .
\end{aligned}
$$

Let $\tilde{g}(k)$ be

$$
\tilde{g}(k):=\frac{(k+a)}{R} e^{-t\left(\frac{k+a}{R}\right)^{2}}
$$

and apply the Poisson resummation formula

$$
\sum_{n \in \mathbb{Z}} \tilde{g}(n)=\sum_{n \in \mathbb{Z}} 2 \pi g(2 \pi n)
$$

Here $g(x)$ is the Fourier transformation of $\tilde{g}(k)$. This $g(x)$ is calculated as

$$
2 \pi g(x)=\int d k e^{i k x} \tilde{g}(k)=\frac{R^{2} \sqrt{\pi}}{2 t^{3 / 2}} i x e^{-i a x} e^{-\frac{R^{2} x^{2}}{4 t}} .
$$

Thus

$$
\sum_{n \in \mathbb{Z}} \tilde{g}(n)=\sum_{n \in \mathbb{Z}} 2 \pi g(2 \pi n)=\sum_{n \in \mathbb{Z}} \frac{R^{2} \sqrt{\pi}}{2 t^{3 / 2}} 2 \pi i n e^{-2 \pi i n a} e^{-\frac{4 \pi^{2} R^{2} n^{2}}{4 t}} .
$$

Substituting this relation to Eq. A27), we obtain

$$
\begin{aligned}
\eta\left(-i D^{1 \mathrm{D}}, s\right) & =\frac{1}{\Gamma\left(\frac{s+1}{2}\right)} \int_{0}^{\infty} d t t^{\frac{s-1}{2}} \sum_{n \in \mathbb{Z}} \frac{R^{2} \sqrt{\pi}}{2 t^{3 / 2}} 2 \pi i n e^{-2 \pi i n a} e^{-\frac{\pi^{2} R^{2} n^{2}}{t}} \\
& =\frac{R^{2} 2 \pi \sqrt{\pi}}{\Gamma\left(\frac{s+1}{2}\right)} \sum_{n=1}^{\infty} n \sin (2 \pi a n) A_{n},
\end{aligned}
$$

where $A_{n}$ is defined and calculated as

$$
A_{n}=\int_{0}^{\infty} d t t^{\frac{s}{2}-2} e^{-\frac{\pi^{2} R^{2} n^{2}}{t}}=\Gamma\left(-\frac{s}{2}+1\right)\left(\pi^{2} R^{2} n^{2}\right)^{\frac{s}{2}-1} .
$$

Then $\eta\left(-i D^{1 \mathrm{D}}, 0\right)$ is calculated as

$$
\eta\left(-i D^{1 \mathrm{D}}, 0\right)=\frac{2}{\pi} \sum_{n=1}^{\infty} \frac{\sin (2 \pi a n)}{n}=1-2(a-[a]),
$$


where the last equality is obtained from the Fourier transformation of the linear function in a range $0<a<1$ and extend it to the whole region, using the periodicity in $a \rightarrow a+$ integers. The limit where $a$ goes to an integer should be taken from the positive side. This result is the same as Eq. A26 as expected.

[1] M. F. Atiyah and I. M. Singer, "The index of elliptic operators on compact manifolds," Bull. Am. Math. Soc. 69, 422 (1963). doi:10.1090/S0002-9904-1963-10957-X

[2] M. F. Atiyah and I. M. Singer, "The Index of elliptic operators. 1," Annals Math. 87, 484 (1968). doi:10.2307/1970715

[3] R. Jackiw and C. Rebbi, "Spinor Analysis of Yang-Mills Theory," Phys. Rev. D 16, 1052 (1977). doi:10.1103/PhysRevD.16.1052

[4] K. Fujikawa, "Path Integral Measure for Gauge Invariant Fermion Theories," Phys. Rev. Lett. 42, 1195 (1979). doi:10.1103/PhysRevLett.42.1195

[5] M. F. Atiyah, V. K. Patodi and I. M. Singer, "Spectral asymmetry and Riemannian Geometry I," Math. Proc. Cambridge Phil. Soc. 77, 43 (1975). doi:10.1017/S0305004100049410

[6] M. F. Atiyah, V. K. Patodi and I. M. Singer, "Spectral asymmetry and Riemannian geometry II,” Math. Proc. Cambridge Phil. Soc. 78, 405 (1975). doi:10.1017/S0305004100051872

[7] M. F. Atiyah, V. K. Patodi and I. M. Singer, "Spectral asymmetry and Riemannian geometry. III," Math. Proc. Cambridge Phil. Soc. 79, 71 (1976). doi:10.1017/S0305004100052105

[8] R. Stora, "Algebraic Structure And Topological Origin Of Anomalies," Progress in Gauge Field Theory. NATO ASI Series (Series B: Physics), vol 115. Springer, Boston, MA. (1984) doi:10.1007/978-1-4757-0280-4_19

[9] B. Zumino, "Chiral Anomalies And Differential Geometry: Lectures Given At Les Houches, August 1983," In *Treiman, S.b. ( Ed.) Et Al.: Current Algebra and Anomalies*, 361-391 and Lawrence Berkeley Lab. - LBL-16747 (83,REC.OCT.) 46p (1985)

[10] B. Zumino, Y. S. Wu and A. Zee, "Chiral Anomalies, Higher Dimensions, and Differential Geometry," Nucl. Phys. B 239, 477 (1984). doi:10.1016/0550-3213(84)90259-1

[11] L. Alvarez-Gaume and P. H. Ginsparg, "The Topological Meaning of Nonabelian Anomalies," Nucl. Phys. B 243, 449 (1984). doi:10.1016/0550-3213(84)90487-5

[12] T. Sumitani, "Chiral Anomalies and the Generalized Index Theorem," J. Phys. A 17, L811 
(1984). doi:10.1088/0305-4470/17/14/016

[13] A. N. Redlich, "Parity Violation and Gauge Noninvariance of the Effective Gauge Field Action in Three-Dimensions," Phys. Rev. D 29, 2366 (1984). doi:10.1103/PhysRevD.29.2366

[14] A. J. Niemi and G. W. Semenoff, "Axial Anomaly Induced Fermion Fractionization and Effective Gauge Theory Actions in Odd Dimensional Space-Times," Phys. Rev. Lett. 51, 2077 (1983). doi:10.1103/PhysRevLett.51.2077

[15] L. Alvarez-Gaume, S. Della Pietra and G. W. Moore, "Anomalies and Odd Dimensions," Annals Phys. 163, 288 (1985). doi:10.1016/0003-4916(85)90383-5

[16] A. Gromov, K. Jensen and A. G. Abanov, "Boundary effective action for quantum Hall states," Phys. Rev. Lett. 116, no. 12, 126802 (2016) doi:10.1103/PhysRevLett.116.126802 [arXiv:1506.07171 [cond-mat.str-el]].

[17] E. Witten, "Fermion Path Integrals And Topological Phases," Rev. Mod. Phys. 88, no. 3, 035001 (2016) doi:10.1103/RevModPhys.88.035001 [arXiv:1508.04715 [cond-mat.mes-hall]].

[18] M. A. Metlitski, "S-duality of $u(1)$ gauge theory with $\theta=\pi$ on non-orientable manifolds: Applications to topological insulators and superconductors," arXiv:1510.05663 [hep-th].

[19] N. Seiberg and E. Witten, "Gapped Boundary Phases of Topological Insulators via Weak Coupling," PTEP 2016, no. 12, 12C101 (2016) doi:10.1093/ptep/ptw083 [arXiv:1602.04251 [cond-mat.str-el]].

[20] Y. Tachikawa and K. Yonekura, "Gauge interactions and topological phases of matter," PTEP 2016, no. 9, 093 B07 (2016) doi:10.1093/ptep/ptw131 [arXiv:1604.06184 [hep-th]].

[21] D. S. Freed and M. J. Hopkins, "Reflection positivity and invertible topological phases," arXiv:1604.06527 [hep-th].

[22] E. Witten, "The 'Parity' Anomaly On An Unorientable Manifold," Phys. Rev. B 94, no. 19, 195150 (2016) doi:10.1103/PhysRevB.94.195150 [arXiv:1605.02391 [hep-th]].

[23] K. Yonekura, "Dai-Freed theorem and topological phases of matter," JHEP 1609, 022 (2016) doi:10.1007/JHEP09(2016)022 [arXiv:1607.01873 [hep-th]].

[24] K. Hasebe, "Higher (Odd) Dimensional Quantum Hall Effect and Extended Dimensional Hierarchy," Nucl. Phys. B 920, 475 (2017) doi:10.1016/j.nuclphysb.2017.03.017 [arXiv:1612.05853 [hep-th]].

[25] Y. Yu, Y. S. Wu and X. Xie, "Bulkedge correspondence, spectral flow and AtiyahPatodiSinger theorem for the Z2 -invariant in topological insulators," Nucl. Phys. B 916, 550 
(2017) doi:10.1016/j.nuclphysb.2017.01.018 [arXiv:1607.02345 [cond-mat.mes-hall]].

[26] D. M. Grabowska and D. B. Kaplan, "Nonperturbative Regulator for Chiral Gauge Theories?," Phys. Rev. Lett. 116, no. 21, 211602 (2016) doi:10.1103/PhysRevLett.116.211602 [arXiv:1511.03649 [hep-lat]].

[27] H. Fukaya, T. Onogi, S. Yamamoto and R. Yamamura, "Six-dimensional regularization of chiral gauge theories," PTEP 2017, no. 3, 033 B06 (2017) doi:10.1093/ptep/ptx017 [arXiv:1607.06174 [hep-th]].

[28] K. i. Okumura and H. Suzuki, "Fermion number anomaly with the fluffy mirror fermion," PTEP 2016, no. 12, 123B07 (2016) doi:10.1093/ptep/ptw167 [arXiv:1608.02217 [hep-lat]].

[29] Y. Hamada and H. Kawai, "Axial U(1) current in Grabowska and Kaplans formulation," PTEP 2017, no. 6, 063 B09 (2017) doi:10.1093/ptep/ptx086 [arXiv:1705.01317 [hep-lat]].

[30] C. G. Callan, Jr. and J. A. Harvey, "Anomalies and Fermion Zero Modes on Strings and Domain Walls," Nucl. Phys. B 250, 427 (1985). doi:10.1016/0550-3213(85)90489-4

[31] D. B. Kaplan, "A Method for simulating chiral fermions on the lattice," Phys. Lett. B 288, 342 (1992) doi:10.1016/0370-2693(92)91112-M [hep-lat/9206013].

[32] Y. Shamir, "Chiral fermions from lattice boundaries," Nucl. Phys. B 406, 90 (1993) doi:10.1016/0550-3213(93)90162-I [hep-lat/9303005].

[33] V. Furman and Y. Shamir, "Axial symmetries in lattice QCD with Kaplan fermions," Nucl. Phys. B 439, 54 (1995) doi:10.1016/0550-3213(95)00031-M [hep-lat/9405004].

[34] E. Witten, “An SU(2) Anomaly," Phys. Lett. 117B, 324 (1982). doi:10.1016/03702693(82)90728-6

[35] M. Hortacsu, K. D. Rothe and B. Schroer, "Zero Energy Eigenstates for the Dirac Boundary Problem," Nucl. Phys. B 171, 530 (1980). doi:10.1016/0550-3213(80)90384-3

[36] M. Luscher, "The Schrodinger functional in lattice QCD with exact chiral symmetry," JHEP 0605, 042 (2006) doi:10.1088/1126-6708/2006/05/042 [hep-lat/0603029].

[37] H. Neuberger, "Exactly massless quarks on the lattice," Phys. Lett. B 417, 141 (1998) doi:10.1016/S0370-2693(97)01368-3 [hep-lat/9707022].

[38] H. Neuberger, "More about exactly massless quarks on the lattice," Phys. Lett. B 427, 353 (1998) doi:10.1016/S0370-2693(98)00355-4 [hep-lat/9801031].

[39] M. Trott, S. Trott and Ch. Schnittler, "Normalization, Orthogonality, and Completeness for the Finite Step Potential," Phys. Stat. Sol. (b) 151, (1989) K123. 
[40] V. N. Marachevsky and D. V. Vassilevich, "Chiral anomaly for local boundary conditions," Nucl. Phys. B 677, 535 (2004) doi:10.1016/j.nuclphysb.2003.11.009 [hep-th/0309019].

[41] C. G. Beneventano and E. M. Santangelo, "Spectral functions of the Dirac operator under local boundary conditions," in Mathematical physics frontiers, edited by C. V. Benton (Nova Science Publishers, Hauppauge, New York, 2004) [hep-th/0405221].

[42] P. Hasenfratz, V. Laliena and F. Niedermayer, "The Index theorem in QCD with a finite cutoff," Phys. Lett. B 427, 125 (1998) doi:10.1016/S0370-2693(98)00315-3 [hep-lat/9801021].

[43] M. F. L. Golterman, K. Jansen and D. B. Kaplan, "Chern-Simons currents and chiral fermions on the lattice," Phys. Lett. B 301, 219 (1993) doi:10.1016/0370-2693(93)90692-B [hep-lat/9209003].

[44] L. Alvarez-Gaume, S. Della Pietra and V. Della Pietra, "The Effective Action for Chiral Fermions," Phys. Lett. B 166, 177 (1986). doi:10.1016/0370-2693(86)91373-0

[45] S. Della Pietra, V. Della Pietra and L. Alvarez-Gaume, "The Chiral Determinant and the $\eta$ Invariant," Commun. Math. Phys. 109, 691 (1987). doi:10.1007/BF01208963

[46] D. B. Kaplan and M. Schmaltz, "Domain wall fermions and the eta invariant," Phys. Lett. B 368, 44 (1996) doi:10.1016/0370-2693(95)01485-3 [hep-th/9510197].

[47] K. Hashimoto, X. Wu and T. Kimura, "Edge states at an intersection of edges of a topological material," Phys. Rev. B 95, no. 16, 165443 (2017) doi:10.1103/PhysRevB.95.165443 [arXiv:1702.00624 [cond-mat.mes-hall]].

[48] T. M. Apostol, "Introduction to Analytic Number Theory," Undergraduate Texts in Mathematics. Springer-Verlag, New York-Heidelberg, 1976. 